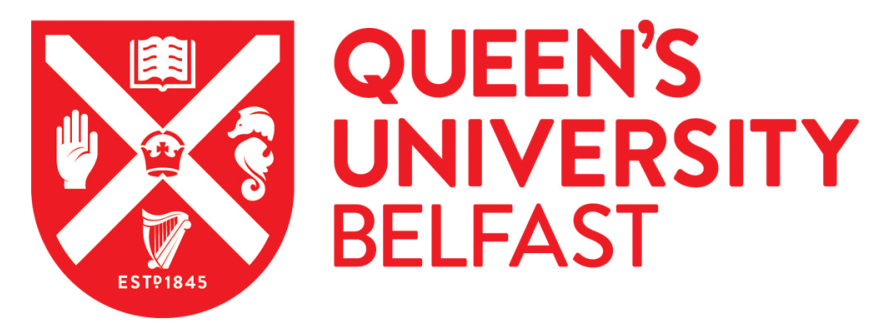

\title{
SNe la from double detonations: Impact of core-shell mixing on the carbon ignition mechanism
}

\author{
Gronow, S., Collins, C., Ohlmann, S. T., Pakmor, R., Kromer, M., Seitenzahl, I. R., Sim, S. A., \& Röpke, F. K. \\ (2020). SNe la from double detonations: Impact of core-shell mixing on the carbon ignition mechanism. \\ Astronomy \& Astrophysics, 635. https://doi.org/10.1051/0004-6361/201936494
}

Published in:

Astronomy \& Astrophysics

Document Version:

Peer reviewed version

Queen's University Belfast - Research Portal:

Link to publication record in Queen's University Belfast Research Portal

Publisher rights

(c) 2020 ESO

This work is made available online in accordance with the publisher's policies. Please refer to any applicable terms of use of the publisher.

\section{General rights}

Copyright for the publications made accessible via the Queen's University Belfast Research Portal is retained by the author(s) and / or other copyright owners and it is a condition of accessing these publications that users recognise and abide by the legal requirements associated with these rights.

Take down policy

The Research Portal is Queen's institutional repository that provides access to Queen's research output. Every effort has been made to ensure that content in the Research Portal does not infringe any person's rights, or applicable UK laws. If you discover content in the Research Portal that you believe breaches copyright or violates any law, please contact openaccess@qub.ac.uk. 


\title{
SNe la from double detonations: Impact of core-shell mixing on the carbon ignition mechanism
}

\author{
Sabrina Gronow ${ }^{1,2, \star}$, Christine Collins ${ }^{3}$, Sebastian T. Ohlmann ${ }^{4,1}$, Rüdiger Pakmor ${ }^{5}$, Markus Kromer ${ }^{1,6}$, Ivo R \\ Seitenzahl ${ }^{7}$, Stuart A. Sim ${ }^{3}$, and Friedrich K. Röpke ${ }^{1,6}$ \\ 1 Heidelberger Institut für Theoretische Studien, Schloss-Wolfsbrunnenweg 35, 69118 Heidelberg, Germany \\ 2 Zentrum für Astronomie der Universität Heidelberg, Astronomisches Rechen-Institut, Mönchhofstr. 12-14, 69120 Heidelberg, Ger- \\ many \\ 3 Astrophysics Research Center, School of Mathematics and Physics, Queen's University Belfast, Belfast BT7 1NN, Northern Ire- \\ land, UK \\ 4 Max Planck Computing and Data Facility, Gießenbachstraße 2, 85748 Garching, Germany \\ 5 Max Planck Institute for Astrophysics, Karl-Schwarzschild-Straße 1, 85748, Garching, Germany \\ 6 Zentrum für Astronomie der Universität Heidelberg, Institut für Theoretische Astrophysik, Philosophenweg 12, 69120 Heidelberg, \\ Germany \\ ${ }^{7}$ School of Science, University of New South Wales, Australian Defence Force Academy, Canberra, ACT 2600, Australia
}

Received August 10, 2019 / Accepted January 23, 2020

\begin{abstract}
Sub-Chandrasekhar mass white dwarfs accreting a helium shell on a carbon-oxygen core are potential progenitors of normal Type Ia supernovae. This work focuses on the details of the onset of the carbon detonation in the double detonation sub-Chandrasekhar model. In order to simulate the influence of core-shell mixing on the carbon ignition mechanism, the helium shell and its detonation are followed with an increased resolution compared to the rest of the star treating the propagation of the detonation wave more accurately. This significantly improves the predictions of the nucleosynthetic yields from the helium burning. The simulations were carried out with the Arepo code. A carbon-oxygen core with a helium shell was set up in one dimension and mapped to three dimensions. We ensured the stability of the white dwarf with a relaxation step before the hydrodynamic detonation simulation started. Synthetic observables were calculated with the radiative transfer code ARTIs. An ignition mechanism of the carbon detonation was observed, which received little attention before. In this "scissors mechanism", the impact the helium detonation wave has on unburnt material when converging opposite to its ignition spot is strong enough to ignite a carbon detonation. This is possible in a carbon enriched transition region between the core and shell. The detonation mechanism is found to be sensitive to details of the core-shell transition and our models illustrate the need to consider core-shell mixing taking place during the accretion process. Even though the detonation ignition mechanism differs form the converging shock mechanism, the differences in the synthetic observables are not significant. Though they do not fit observations better than previous simulations, they illustrate the need for multi-dimensional simulations.
\end{abstract}

Key words. Hydrodynamics - Methods: numerical - Nuclear reactions, nucleosynthesis, abundances - Radiative transfer - supernovae: general - white dwarfs

\section{Introduction}

The progenitor evolution and the conditions that lead to the onset of explosions of Type Ia supernovae (SNe Ia) are highly controversial. Recent results (e.g., Gilfanov \& Bogdán 2010; Sim et al. 2013) indicate that the majority of events cannot be easily explained with the long-time favored model of thermonuclear explosions in Chandrasekhar-mass white dwarfs (WDs) (Arnett 1969; Reinecke et al. 2002; Seitenzahl et al. 2013b, but also see Seitenzahl et al. 2013a). A promising alternative is a thermonuclear carbon-oxygen (CO) detonation in sub-Chandrasekhar mass WDs (Shigeyama et al. 1992; Sim et al. 2010; Shen et al. 2018; Wilk et al. 2018), but the mechanism by which it is initiated is not fully understood. Violent mergers of two white dwarfs have been suggested (e.g., Iben \& Tutukov 1984; Guillochon et al. 2010; Pakmor et al.

^ e-mail: sabrina.gronow@h-its.org, Fellow of the International Max Planck Research School for Astronomy and Cosmic Physics at the Heidelberg University (IMPRS-HD)
2010, 2011, 2013). As an alternative to that, Nomoto (1982), Woosley \& Weaver (1994), Bildsten et al. (2007), and Kromer et al. (2010) investigated sub-Chandrasekhar mass white dwarfs in binaries. In this case a white dwarf accretes helium $(\mathrm{He})$ from a companion and ignition conditions arise. Recent reviews on the different progenitor systems are Maoz et al. (2014) and Wang et al. (2012).

Here we consider a sub-Chandrasekhar mass white dwarf as a progenitor that explodes in the double detonation scenario. In this scenario, a carbon-oxygen WD has accreted a rather massive helium shell from a helium white dwarf. A detonation is ignited at the base of the shell when critical conditions are reached through thermal instability. Following the detonation in the shell, three main scenarios have been suggested so far: First, the He shell detonation directly triggers a second detonation at the interface between the He shell and $\mathrm{CO}$ core, which is referred to as the edge-lit scenario (e.g., Livne \& Glasner 1990; Sim et al. 2012). Second, a shock wave that is driven by the helium shell 
detonation propagates into the core and converges spherically thus igniting a second detonation, which is referred to as the converging shock scenario (e.g., Livne 1990; Livne \& Glasner 1991; Livne \& Arnett 1995; Fink et al. 2007, 2010; Moll \& Woosley 2013; Shen \& Bildsten 2014). Third, no secondary core detonation is ignited and a faint .Ia supernova ensues (e.g., Bildsten et al. 2007; Waldman et al. 2011; Sim et al. 2012).

The converging shock scenario corresponds to the classical double detonation scenario. In the context of this scenario three questions remain open; (1) how does the helium detonation in the shell form, (2) how is the core detonation initiated, and (3) what are the yields of the helium detonation and their effects on the spectra and light curves.

Previous work by Kromer et al. (2010), Boyle et al. (2017), and Botyánszki et al. (2018), for example, points out that the synthetic spectra of the sub-Chandrasekhar mass models are too red. Further He is not present in the observed spectra. Therefore the mass of the He shell must be small. Townsley et al. (2019) show that a double detonation of a $\mathrm{CO}$ white dwarf with a less massive He shell is possible. Their model is modestly enriched with $\mathrm{C}$ which supports burning to heavier elements during the He detonation. Compared to their work we now increase the resolution in the He shell and use a different numerical approach.

Our simulations focus on the last two questions mentioned above. We study the influence of the burning products of the helium shell on the ejecta composition and the details of igniting a detonation in the $\mathrm{CO}$ core. Radiative transfer calculations allow the discussion of their impact on the observables. Relating to question (1), Glasner et al. (2018) investigated whether a helium detonation can ignite in the He shell. Röpke et al. (2007) and Seitenzahl et al. (2009) studied conditions for carbon detonation ignition and the processes leading to it were simulated by Fink et al. (2007, 2010) and Moll \& Woosley (2013), addressing question (2). The effect of the He shell ejecta on the observables was discussed by Kromer et al. (2010) and Townsley et al. (2012, 2019), corresponding to question (3).

Previous work was mostly carried out in one or two spatial dimensions (1D and 2D). Moll \& Woosley (2013) perform threedimensional (3D) simulations of one quarter of the white dwarf assuming two synchronous spherical detonators and using mirror symmetry. García-Senz et al. (2018) carry out 3D simulations of rigidly rotating sub-Chandrasekhar mass white dwarfs with a smoothed particle hydrodynamics code. We follow up on such studies and present grid-based hydrodynamic simulations comprising a whole (non-rotating) white dwarf to check whether a detonation in a helium shell can trigger a second detonation in the core leading to a complete incineration of the white dwarf and a supernova explosion. The use of the moving mesh code ArePo (Springel 2010) allows a better resolution of the helium shell and a more accurate simulation of the propagation of the detonation front in the helium shell compared to previous simulations by others (e.g., Fink et al. 2007; Moll \& Woosley 2013). In our simulations we observe a mechanism for igniting the secondary core detonation that previously received little attention. The convergence of the helium detonation wave on the far side of the ignition spot causes a second detonation at the edge of the $\mathrm{CO}$ core. This detonation propagates through the whole core disrupting the white dwarf. Livne \& Arnett (1995) and García-Senz et al. (1999) mention a delayed edge-lit detonation with a second detonation forming at the antipode of the He ignition point, and Forcada (2008) presents a simulation showing this effect. A detailed discussion of the progenitor and explosion mechanism, however, is missing in these publications. The same detonation ignition mechanism is described in García-Senz et al. (2018) for a rotating white dwarf. Following up on their work we explain the ignition mechanism in detail and discuss its effect on observables in the analyzes of multi-dimensional radiative transfer calculations.

The methods are described in Section 2 and details on the models are presented in Section 3. Section 4 discusses the results from the hydrodynamic simulations and their significance in the framework of previous work. Radiative transfer calculations are presented in Section 5. We conclude in Section 6. The simulation data of all our models will be made available on the Heidelberg Supernova Model Archive (HESMA, Kromer et al. 2017).

\section{Methods}

\subsection{Hydrodynamics}

Three dimensional simulations are carried out using the moving mesh code Arepo (Springel 2010). The code is based on a Voronoi tessellation of space with mesh generating points moving along with the hydrodynamic flow. This leads to a nearly Lagrangian scheme. A second-order finite-volume method is employed to solve the Euler-Poisson equations of hydrodynamics with tree-based self-gravity as a source term. The Godunov method is used as described in Springel (2010) with the improved scheme of Pakmor et al. (2016). For modeling reactive flows, a source term is added to the energy equation and balance equations for nuclear species are followed. To this end, the nuclear network solver of Pakmor et al. (2012) is coupled to the hydrodynamic solver of the Arepo code (Pakmor et al. 2013). If not stated otherwise, we employ a nuclear network consisting of 33 species comprising $\mathrm{n}, \mathrm{p},{ }^{4} \mathrm{He},{ }^{12} \mathrm{C},{ }^{13} \mathrm{~N},{ }^{16} \mathrm{O},{ }^{20} \mathrm{Ne},{ }^{22} \mathrm{Na}$, ${ }^{23} \mathrm{Na},{ }^{24} \mathrm{Mg},{ }^{25} \mathrm{Mg},{ }^{26} \mathrm{Mg},{ }^{27} \mathrm{Al},{ }^{28} \mathrm{Si},{ }^{29} \mathrm{Si},{ }^{30} \mathrm{Si},{ }^{31} \mathrm{P},{ }^{32} \mathrm{~S},{ }^{36} \mathrm{Ar}$, ${ }^{40} \mathrm{Ca},{ }^{44} \mathrm{Ti},{ }^{45} \mathrm{Ti},{ }^{46} \mathrm{Ti},{ }^{47} \mathrm{~V},{ }^{48} \mathrm{Cr},{ }^{49} \mathrm{Cr},{ }^{50} \mathrm{Cr},{ }^{51} \mathrm{Mn},{ }^{52} \mathrm{Fe},{ }^{53} \mathrm{Fe}$, ${ }^{54} \mathrm{Fe},{ }^{55} \mathrm{Co}$, and ${ }^{56} \mathrm{Ni}$.

Following Fryxell et al. (1989) and Appendix A of Townsley et al. (2016), burning is disabled when the conditions

$\boldsymbol{\nabla} \cdot \boldsymbol{v}<0$ and $\nabla P \cdot \frac{r_{\text {cell }}}{P_{\text {cell }}}>0.66$

indicate that the corresponding region is located inside the shock. The Helmholtz equation of state was implemented by Pakmor et al. (2013) based on Timmes \& Swesty (2000) and closes the system of equations to be solved.

The adaptive mesh refinement capability of AREPo allows us to better resolve the helium shell and the propagation of the detonation wave within it. We employ an additional refinement in two different regions: the He shell and the location of the carbon detonation ignition (see Sec. 3.3 for the coordinates). A passive scalar is used to track the location of the He shell. This is needed as $\mathrm{He}$ is not only present in the shell, but also in the background of the white dwarf.

The mass of a cell is chosen as the refinement criterion. Similar to Pakmor et al. (2013) an explicit refinement is used for a better mass resolution and a reference mass for a cell is fixed. If a cell mass exceeds this reference mass by a factor of two the cell is split.

\subsection{Nucleosynthesis postprocessing}

As stated above a 33 isotope nuclear network is used to follow the nucleosynthesis in the hydrodynamics simulation. This gives a good approximation to the final abundances and the energy release during the explosion. To determine detailed yields, nucleosynthesis postprocessing is carried out in a subsequent step 
based on two million tracer particles that are placed into the hydrodynamic explosion model. These sample the initial mass distribution, each representing a mass of about $1 \times 10^{27} \mathrm{~g}$ of material whose temperature and density evolution is tracked (Travaglio et al. 2004).

The thermodynamic tracer particle trajectories form the basis of the postprocessing nucleosynthesis calculation with a network involving 384 isotopes (Pakmor et al. 2012). It reaches from neutrons to ${ }^{98} \mathrm{Mo}$. The reaction rates are taken from the REACLIB data base (Rauscher \& Thielemann 2000) as in Pakmor et al. (2012). The hydrodynamic simulation extends up to $100 \mathrm{~s}$ after ignition. By this time, homologous expansion is reached to a good approximation while the nuclear burning is already complete after a few seconds. With its two million tracer particles, the postprocessing step determines the three-dimensional chemical composition of the ejecta with sufficient accuracy for subsequent radiative transfer calculations.

\subsection{Radiative transfer}

The time-dependent multi-dimensional Monte Carlo radiative transfer code Artis (Sim 2007; Kromer \& Sim 2009, based on the methods of Lucy 2002, 2003, 2005) is used to derive synthetic observables for the models. The postprocessing abundances and final ejecta density are mapped onto a $50^{3}$ Cartesian grid using the scheme described by Fink et al. (2014, see also Kromer et al. 2010). In each radiative transfer simulation $2.56 \times 10^{7}$ energy packets are tracked as they propagate through the ejecta for 111 logarithmically spaced time steps between 2 and 120 days after the explosion. We use the atomic data set as described by Gall et al. (2012), adopt a gray approximation in cells that are optically thick (cf. Kromer \& Sim 2009), and assume local thermodynamic equilibrium for the first ten time steps (times $<3$ days after explosion). Line-of-sight dependent light curves are calculated as by Kromer et al. (2010). The escaping photons are binned into a grid of ten equal solid-angle bins in $\mu=\cos \theta$ where $\theta$ is the angle between the line of sight and the $\mathrm{z}-$ axis of the model. The line-of-sight dependent spectra are calculated using "virtual-packets" as described by Bulla et al. (2015). Where an energy packet interaction occurs, a virtual-packet is created with frequency and energy equal to the energy packet at the point of creation. The virtual-packet is propagated toward a predefined observer direction $n_{\mathrm{obs}}$, and contributes to the emergent spectrum. This approach significantly reduces the Monte Carlo noise in the angle dependent spectra.

\section{Models}

\subsection{Model setup}

Nine hydrodynamics simulations were carried out following the evolution of a white dwarf that consists of a carbon-oxygen core and a helium shell. Carbon and oxygen make up $50 \%$ by mass, respectively, of the core material. In our initial models, the composition changes in a small transition region at the edge of the core to pure helium. The details of the models are listed in Table 1 . Their total masses of $0.91 \mathrm{M}_{\odot}(\mathrm{M} 3 \mathrm{a})$ and $1.05 \mathrm{M}_{\odot}(\mathrm{M} 1$ and M2) are chosen to be similar to Models 1 and 3 (hereafter FM1 and FM3) in Fink et al. (2010). Model M2a is our reference model. Modifications of the base setup are made to study the effect of different parameters. The different models explore the influence of the mixing of carbon into the shell (M1a and M2a), the white dwarf mass (M2a and M3a), the resolution (M2a, M2a_13, M2a_21, M2a_36, and M2a_79), the nuclear network in the hy-
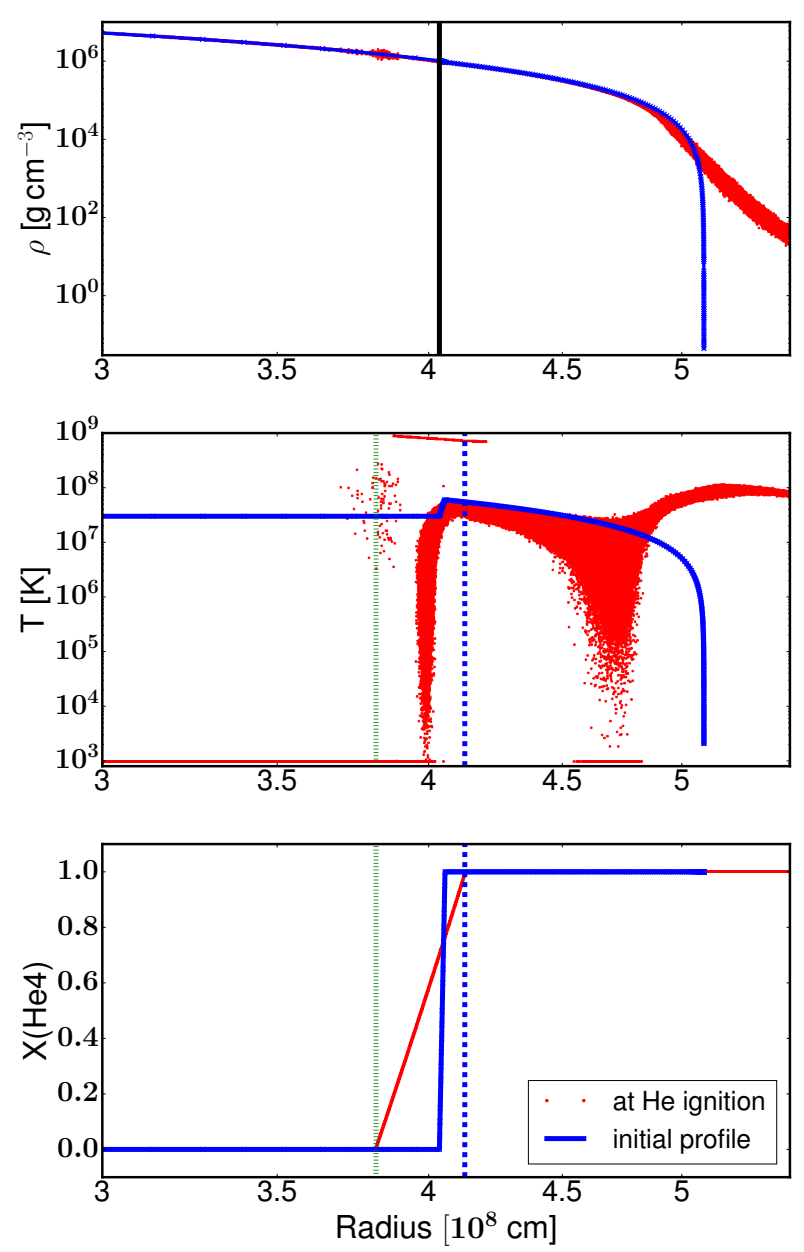

Fig. 1: Radial profile of the density, temperature, and helium mass fraction in an interval of 3 to $5.5 \times 10^{8} \mathrm{~cm}$ of the initial setup and at helium ignition of Model M2a; the black solid, green dotted, and blue dashed lines represent the core-shell transition, base of the helium shell, and outer edge of the transition region, respectively; the cells with temperatures higher than $7 \times 10^{8} \mathrm{~K}$ in the profile at He ignition represent the detonating cells of the He detonation.

drodynamic calculations (M2a and M2a_i55), and the He ignition setup (M2a and b; see Table 1). The reference model M2a is discussed in Sections 3.2 and 3.3 as well as Sections 4.1 and 4.2. A comparison and discussion of the hydrodynamic simulations in the context of Models M1a and M2b to M3a follows in Sections 4.3 to 4.6 .

The white dwarf was set up in 1D by integrating the equations of hydrostatic equilibrium. The total mass of the white dwarf $\left(M_{\text {tot }}\right)$ and the transition density $\left(\rho_{\mathrm{S}}\right)$ at which the helium shell begins are initial parameters. The position at which the helium shell begins as well as its mass depend on these parameters. The temperature was set to be constant within the core $\left(T_{\mathrm{C}}\right)$ while $T_{\mathrm{S}}$ describes the temperature at the outer edge of the transition between core and shell beyond which it declines adiabatically. The helium shell mass was not set explicitly but was determined by an iteration based on the fixed total mass of the WD and density at the base of the helium shell, $\rho_{\mathrm{S}}$, while the central density $\rho_{\mathrm{C}}$ is variable. 
Fig. 1 shows the initial setup of Model M2a in the radial range from 3 to $5.5 \times 10^{8} \mathrm{~cm}$ (in blue). The model parameters are listed in Table 1 . The jump in the temperature profile (middle row) and helium mass fraction (bottom row) indicates the location of the change from the CO core to the helium shell of the WD. The vertical line in the density profile marks the position of $\rho_{S}$ corresponding to the other profiles. The core radius is at about $4.0 \times 10^{8} \mathrm{~cm}$. The transition between core and shell is set to only consist of 20 cells in the 1D setup following a linear trend in the temperature and abundances. This transition region extends from $4.039 \times 10^{8} \mathrm{~cm}$ to $4.058 \times 10^{8} \mathrm{~cm}$ in radius. Based on the setup in $1 \mathrm{D}$, the model of a white dwarf with a helium shell is mapped to 3D using the HEALPix method (Górski et al. 2005) on concentric shells according to Ohlmann et al. (2017).

\subsection{Relaxation}

After mapping onto the 3D grid of AREPo, a relaxation step following Ohlmann et al. (2017) was carried out to eliminate spurious velocities that can be caused by discrepancies between the pressure gradient and gravity. Earlier work by, for example, Fink et al. (2007) performed the explosion simulations without prior relaxation of the star; however, when mapping the initial WD structure onto the unstructured computational mesh of AREPO, relaxation is necessary to obtain a hydrostatic equilibrium. The white dwarf was relaxed in a hydrodynamic simulation with the nuclear reactions switched off for ten dynamical time scales, which are defined here by the sound crossing time,

$\tau_{\text {dyn }}=\int_{0}^{R} \frac{\mathrm{d} r}{v_{s}}$,

with radius $R$ and local sound speed $v_{s}$, which, because the density is not constant, varies with radius. In the relaxation process the velocities were damped until $80 \%$ of the relaxation time has passed. The simulation was continued for two dynamical timescales without damping to verify stability of the model. The conditions for stability posed in Ohlmann et al. (2017) are fulfilled for all models presented in this paper.

During the relaxation process mixing took place between the helium shell and CO core washing out the interface between them. This mixing was additionally enhanced after the relaxation for all models except M1a and M3a. We therefore identify the shell with material in which the helium mass fraction exceeds 0.01. Naturally, this shifts the original core and shell masses and the values for the post-relaxation helium shell masses are included in Table 1 . They differ by about $0.02 \mathrm{M}_{\odot}$ from the initial setup. $0.01 \mathrm{M}_{\odot}$ of each carbon and oxygen are mixed into the shell.

The effect of mixing during the relaxation phase is illustrated in Fig. 1. The red points represent the radial profiles obtained from the AREPo model at the time of helium ignition after relaxation while the initial 1D model is shown in blue. The core radius shifts inwards to about $3.8 \times 10^{8} \mathrm{~cm}$ and the transition region between core and pure shell material increases in size (see bottom panel in Fig. 1). The radius of the outer end of the transition region shifts outwards due to the additional artificial mixing. The temperature profile (middle panel) shows a similar shift corresponding to the helium mass fraction. However, the transition region does not show an equally broad increase in the temperature. Some cells in the radial interval $\left[3.5 \times 10^{8}, 3.9 \times 10^{8}\right] \mathrm{cm}$ experience an increase in temperature during the relaxation. This does not influence the detonation as the values are too low for ignition of a detonation in the material.

\subsection{Detonation}

In contrast to Pakmor et al. (2013) who model a white dwarf merger, the first detonation in the He shell was not ignited dynamically but it was assumed to be triggered by thermal instability. Glasner et al. (2018) investigate whether such a detonation in the helium shell can develop naturally following the accretion of helium onto the white dwarf and find this to be likely from their simulations. Jacobs et al. (2016) carry out 3D simulations of one eighth of a WD using a low-Mach number code. They conclude that a localized runaway can be achieved taking 3D convection into account. Similar to Livne (1997), Fink et al. (2010), and Woosley \& Kasen (2011), we triggered the helium detonation artificially at the base of the helium shell. This is achieved by increasing the specific thermal energy of selected cells to $5 \times 10^{16} \mathrm{erg} \mathrm{g}^{-1}$. This value was chosen to ignite explosive burning without reaching a non-physical high thermal energy. Comparable values are found by Glasner et al. (2018) in their He detonation ignition simulations.

Due to the mixing the transition region between core and shell has widened. The igniting cells were therefore chosen to enclose a volume of radius $\Delta R$ centered at the outer edge of the transition region. $\Delta R$ was defined as 0.04 times the distance of the central detonating cell to the center of the white dwarf. This value leads to a detonation ignition in a volume similar to that found by Glasner et al. (2018). This construction formally results in a symmetric detonation ignition volume around one point. In the numerical implementation, however, asymmetries are possible due to the Voronoi structure of the grid. Averaged global spherical symmetry (neglecting rotation) allows us to select the detonation point arbitrarily on a sphere corresponding to the base of the helium shell. In our models, it was placed on the positive $z$-axis, that is at $x=y=0$.

The cells in which the helium was ignited can be identified in the temperature profile of Fig. 1. They are located at radii between $3.88 \times 10^{8} \mathrm{~cm}$ and $4.11 \times 10^{8} \mathrm{~cm}$ and have temperatures of at least $7 \times 10^{8} \mathrm{~K}$. The central ignition spot is at a radius of $4.04 \times 10^{8} \mathrm{~cm}$ for Model M2a.

Different initial positions for the helium detonation were tested for Model M2. The detonation in the helium shell was ignited uniformly around one point at the very base of the He shell $\left(R_{\mathrm{C}}, \mathrm{M} 2 \mathrm{~b}\right.$ with core radius $R_{\mathrm{C}}$ after relaxation, green dotted line in Fig. 1), and at the base of the He shell where the peak in the temperature profile is located at the outer edge of the transition region (M2a, blue dashed line in Fig. 1). The radii of the central ignition spots are $4.04 \times 10^{8} \mathrm{~cm}$ and $3.77 \times 10^{8} \mathrm{~cm}$ for Models M2a and b, respectively. A sketch of the ignition spots is shown in Fig. 2. The red curve represents the outer edge of the transition region between core and shell.

As described in Section 2.1 an additional mesh refinement was imposed in the helium shell to better resolve the propagation of the detonation wave. A passive scalar was used to follow the shell material and to determine where the refinement is increased. As we discuss in Sec. 4, Model M2a shows a high increase of the density and temperature in the convergence point of the He detonation wave at the antipode of the helium ignition which triggers a carbon detonation. A further mesh refinement region was added for the location of this second, carbon detonation. This results in two regions with additional refinement over the base resolution: one in the helium shell and another around the helium detonation convergence spot opposite from its ignition. Additional simulations were carried out with different resolutions to check for the convergence of the helium detonation and the carbon detonation. These are Models M2a_79, M2a_36, 
Table 1: Overview of model parameters.

\begin{tabular}{lrrrrrrrrr}
\hline \hline Model & $\begin{array}{r}M_{\text {tot }} \\
{\left[\mathrm{M}_{\odot}\right]}\end{array}$ & $\begin{array}{r}M_{\mathrm{iHeS}} \\
{\left[\mathrm{M}_{\odot}\right]}\end{array}$ & $\begin{array}{r}M_{\mathrm{pHeS}} \\
{\left[\mathrm{M}_{\odot}\right]}\end{array}$ & $\begin{array}{r}T_{\mathrm{S}} \\
{\left[10^{7} \mathrm{~K}\right]}\end{array}$ & $\begin{array}{r}T_{\mathrm{C}} \\
{\left[10^{7} \mathrm{~K}\right]}\end{array}$ & $\begin{array}{r}\rho_{\mathrm{S}} \\
{\left[10^{6} \mathrm{~g} \mathrm{~cm}^{-3}\right]}\end{array}$ & $\begin{array}{r}\rho_{\mathrm{C}} \\
{\left[10^{7} \mathrm{~g} \mathrm{~cm}^{-3}\right]}\end{array}$ & $\begin{array}{r}\text { resolution } \\
{\left[10^{-8} M_{\odot}\right]}\end{array}$ & \# isotopes \\
\hline M1a & 1.05 & 0.051 & 0.064 & 6 & 3 & 1.2 & 4.8 & 3.33 & ignition spot \\
M2a & 1.05 & 0.051 & 0.073 & 6 & 3 & 1.2 & 4.8 & 3.35 & 33 \\
M2b & 1.05 & 0.051 & 0.073 & 6 & 3 & 1.2 & 4.8 & 3.35 & 33 \\
M2a_79 & 1.05 & 0.051 & 0.073 & 6 & 3 & 1.2 & 4.8 & 79.18 & 33 \\
M2a_36 & 1.05 & 0.051 & 0.073 & 6 & 3 & 1.2 & 4.8 & 36.27 & 33 \\
M2a_21 & 1.05 & 0.051 & 0.073 & 6 & 3 & 1.2 & 4.8 & 21.44 & 33 \\
M2a_13 & 1.05 & 0.051 & 0.073 & 6 & 3 & 1.2 & 4.8 & 12.71 & $\mathrm{a}$ \\
M2a_i55 & 1.05 & 0.051 & 0.073 & 6 & 3 & 1.2 & 4.8 & 3.35 & $\mathrm{a}$ \\
M3a & 0.91 & 0.135 & 0.155 & 6 & 3 & 1.5 & 1.9 & 2.76 & 5 \\
\hline
\end{tabular}

Notes. The total mass of the white dwarf $M_{\mathrm{tot}}$, initial mass of the helium shell $M_{\mathrm{iHes}}$ and post-relaxation $M_{\mathrm{pHeS}}$, temperature $T_{\mathrm{S}}$ at the base of the helium shell, core temperature $T_{\mathrm{C}}$, transition density to the shell $\rho_{\mathrm{S}}$, and central density $\rho_{\mathrm{C}}$ are given. Models M2 denote models with an additional mixing of carbon into the He shell after relaxation. The resolution is given for the volume where the detonation wave converges at the antipodes which is the region with the highest level of refinement in Model M2a.

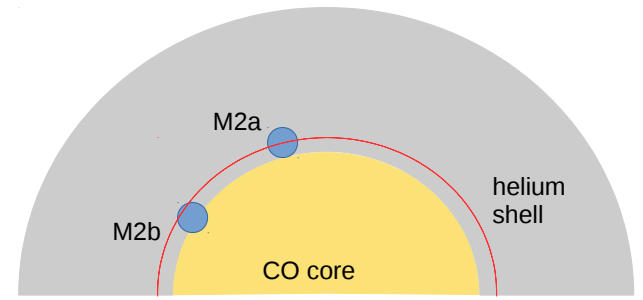

Fig. 2: Schematic illustration of different ignition spots for Model M2 with the white dwarf CO core in yellow and helium shell in gray; symmetric around the base of the shell (M2b) and symmetric around the point of peak temperature (M2a).

M2a_21, and M2a_13. Contrary to simulation M2a and other models no additional refinement is used for Model M2a_79.

In AREPo, refinement is enforced by reducing the reference mass in the respective region. As a standard, a reference mass $M_{\mathrm{R}}$ of $2 \times 10^{27} \mathrm{~g}$ is chosen in regions with base resolution. With each level of refinement the reference mass is decreased. The reference mass is $4 \times 10^{26} \mathrm{~g}$ and $2 \times 10^{26} \mathrm{~g}$ for Models M2a_36 and M2a_21, respectively, which have an additional refinement in the helium shell. The refinement around the convergence spot of the helium detonation is imposed in the region enclosed in $-2 \times$ $10^{8} \mathrm{~cm}<\mathrm{x}<2 \times 10^{8} \mathrm{~cm},-2 \times 10^{8} \mathrm{~cm}<\mathrm{y}<2 \times 10^{8} \mathrm{~cm}$, and $-7 \times$ $10^{8} \mathrm{~cm}<\mathrm{z}<-3 \times 10^{8} \mathrm{~cm}$. The limits of this refinement region are chosen after the first detection of the carbon detonation at the convergence spot. Models M2a_13 and M2a have a reference mass of $1.2 \times 10^{26} \mathrm{~g}$ and $2 \times 10^{25} \mathrm{~g}$, respectively.

\section{Results from the hydrodynamic explosion simulations}

We summarize the results from the hydrodynamic explosion simulations in this section focusing on our reference model M2a for most parts. The detonation ignition mechanism is described in detail followed by an analysis of the final abundances. A discussion of different parameters such as the resolution, ignition spot, and white dwarf mass is carried out. A comparison to previous work concludes this section.

We describe the evolution for our reference model M2a first. After the He detonation is initiated, a detonation wave propagates through the helium shell and a shock wave develops and propagates through the core of the white dwarf. The simulation follows the evolution for $100 \mathrm{~s}$. In Model M2a a volume consisting of 4514 cells is set to detonate initially in the helium detonation.

The propagation of the detonation wave is visible in Fig. 3. It shows the evolution of the carbon mass fraction, temperature, and density (from left to right) at four different times.

The top row illustrates the ignition of the helium detonation in the high temperature spot. The location of the core-shell transition can be inferred from the carbon abundance. The second row shows the propagation of the detonation wave in the helium shell and the shock in the $\mathrm{CO}$ core. The burning in the helium shell is visible as some of the carbon in the core-shell transition region is burned together with the helium. At the same time the energy release leads to a temperature increase. The shock wave driven into the core by the helium detonation is visible in the density slice. The third row of Fig. 3 shows the instant when the detonation wave that propagated through the He shell converges into a spot on the far side of the ignition. This initiates a second detonation in the carbon-oxygen core $1.123 \mathrm{~s}$ after helium ignition. Details of this detonation formation are described in Section 4.1. After the convergence of the He detonation wave and ignition of a carbon detonation, the detonation wave moves inwards with a velocity of about $13.1 \times 10^{8} \mathrm{~cm} \mathrm{~s}^{-1}$ and a detonation wave develops in the core. The carbon detonation wave incinerates the core, thereby running over the shock wave still propagating through the core material. The bottom row in Fig. 3 illustrates this situation: The shock wave sent into the core from the shell detonation is about to converge while the detonation overruns it.

Small asymmetries are visible in the bottom row of Fig. 3 which persist for the remaining simulation time until homologous expansion sets in. These asymmetries might be caused by the irregularity of the grid or the initial asymmetric initiation of the detonation in the helium shell. The Voronoi mesh consists of cells that have different sizes and shapes. Only the cell mass is a 
constant parameter. This grid does not allow a perfect representation of all shapes. The characteristic of the mesh also results in small asymmetries in the initial artificial detonation spot in the helium shell as discussed in Section 3.3. Due to this, the propagation of the detonation wave in the helium shell might differ slightly between different sides of the white dwarf.

\subsection{CO detonation ignition mechanism}

The detonation ignition mechanism shown in Fig. 3 has not been investigated in detail in previous work, which mostly focused on the converging shock mechanism as the possible trigger for the second detonation. It has, however, been briefly described by Livne \& Arnett (1995), García-Senz et al. (1999), Forcada (2008), and García-Senz et al. (2018). We call this mechanism the scissors mechanism because the detonation forms as a shock wave propagates into dense core material while sliding over each other, much like closing scissors.

Fig. 4 shows the temperature in a zoom-in (in time and space) into the region of $-2.5 \times 10^{8} \mathrm{~cm}<\mathrm{x}<2.5 \times 10^{8} \mathrm{~cm}$ and $-6.5 \times 10^{8} \mathrm{~cm}<\mathrm{z}<-2.5 \times 10^{8} \mathrm{~cm}$ of Fig. 3 at $1.080 \mathrm{~s}$ to $1.187 \mathrm{~s}$ after the first detonation ignition. At the helium detonation front the temperature is high with values of about $3.6 \times 10^{9} \mathrm{~K}$. It closes up $1.123 \mathrm{~s}$ after its ignition on the far side of the WD star (see the center panel of Fig. 4). A comparison of the profile of the carbon abundance and temperature in the third row from the top in Fig. 3 shows that the point of convergence is close to the base of the helium shell. This region contains carbon which was mixed into the shell during the relaxation and acts as fuel that can be burned. Peak temperatures of about $2.7 \times 10^{9} \mathrm{~K}$ are reached. Temperature spikes of at least $2.4 \times 10^{9} \mathrm{~K}$ lead to explosive burning in a few cells. These cells each have a volume of about $3.22 \times 10^{19} \mathrm{~cm}^{3}$ on average (corresponding to a radius of about $20 \mathrm{~km}$ assuming a spherical structure of the cell) with a density higher than $3.0 \times 10^{6} \mathrm{~g} \mathrm{~cm}^{-3}$ after $1.123 \mathrm{~s}$. In all detonating cells, the temperature increases further to above $2.8 \times 10^{9} \mathrm{~K} 1.126 \mathrm{~s}$ after the first ignition in the helium shell increasing the robustness of the detonation. The detonating cells have abundances of at least 0.20 in carbon and 0.42 in oxygen, confirming the carbon detonation.

Even in our highest-resolution simulation, the ignition of the carbon detonation is far from being resolved. The detonating cells in the simulation with the highest resolution have a radial extent of about $20 \mathrm{~km}$. Katz \& Zingale (2019) in comparison show that a resolution lower than $1 \mathrm{~km}$ is necessary which can not be reached easily in full $3 \mathrm{D}$ simulations. The detonation in our simulations is at least in parts due to numerical effects. We therefore compare with off-line detonation ignition studies to determine whether a detonation can form physically under the conditions we observe in our simulations. The values of temperature and density are high enough to trigger a detonation according to both Röpke et al. (2007) and Seitenzahl et al. (2009) who determined critical values and sizes for detonation ignition. Röpke et al. (2007) list a temperature of $2.3 \times 10^{9} \mathrm{~K}$ to be sufficient on a $100 \mathrm{~km}$ scale with a density of at least $1.41 \times 10^{6} \mathrm{~g} \mathrm{~cm}^{-3}$. Seitenzahl et al. (2009) give values of at least $5.0 \times 10^{6} \mathrm{~g} \mathrm{~cm}^{-3}$ and $2.0 \times 10^{9} \mathrm{~K}$ for the density and temperature, respectively. We therefore conclude that a physical detonation ignition at the points singled out in our simulation is likely to occur.

\subsection{Final abundances}

The final abundances of ${ }^{4} \mathrm{He},{ }^{12} \mathrm{C},{ }^{16} \mathrm{O},{ }^{28} \mathrm{Si},{ }^{32} \mathrm{~S},{ }^{40} \mathrm{Ca},{ }^{44} \mathrm{Ti}$, and ${ }^{56} \mathrm{Ni}$ in the ejected material as determined from nucleosynthetic postprocessing of the tracer particles (see Section 2.2) are given in Table 2 for Models M1a, FM3, M2a, and M2a_i55. The four left columns list the abundances from the helium detonation, while the four right columns give the final abundances of the core detonation when homologous expansion occurs. The tracer particle approach allows us to separate the yields of the helium detonation from the yields of the core detonation: we identify tracers that are affected by the helium shell detonation based on whether it had a helium mass fraction of at least 0.01 at the beginning of the hydrodynamic detonation simulation. Fink et al. (2010) do not consider the possibility of the ignition of a detonation at the point where the detonation wave converges at the shell-core interface for Model FM3, but they focus on a second detonation that might be ignited in the $\mathrm{CO}$ core due to spherical shock collimation near the center. Their abundances are included in Table 2 for comparison and taken from Fink et al. (2010) and Kromer et al. (2010) for Model FM3.

In Model M2a about equal parts of ${ }^{32} \mathrm{~S}$ and ${ }^{40} \mathrm{Ca}$ are produced in the He shell detonation $\left(\sim 10^{-3} \mathrm{M}_{\odot}\right)$ while the amounts of produced ${ }^{16} \mathrm{O}$ and ${ }^{28} \mathrm{Si}$ are slightly higher. The ${ }^{44} \mathrm{Ti}$ abundance is comparatively low with $7.0 \times 10^{-4} \mathrm{M}_{\odot}$. The ${ }^{12} \mathrm{C}$ is also low - in this case because high enough temperatures are reached to burn to heavier elements. The relatively high amount of helium in the shell detonation is a result of the expansion of the matter during the burning. The density decreases, it cools down and burning stops.

The high temperatures and densities together with carbon in the helium shell allow the production of more heavy elements such as ${ }^{56} \mathrm{Ni}$ compared to Model FM3. The $\alpha$-captures are accelerated due to the presence of ${ }^{12} \mathrm{C}$ (see Fink et al. (2010) for a detailed discussion). There is a difference of about one order of magnitude in most species produced in the helium detonation between our reference model M2a and FM3. Only the ${ }^{12} \mathrm{C}$ and ${ }^{40} \mathrm{Ca}$ abundances are about the same with $1.0 \times 10^{-4} \mathrm{M}_{\odot}$ and $2.2 \times 10^{-4} \mathrm{M}_{\odot}$, and $3.6 \times 10^{-3} \mathrm{M}_{\odot}$ and $2.2 \times 10^{-3} \mathrm{M}_{\odot}$, respectively. The ${ }^{44} \mathrm{Ti}$ abundance shows a reduction by about a factor of five compared to the value found by Fink et al. (2010). In Model M2a we burn about $50 \%$ of the initial helium. This is higher than in Fink et al. (2010) and can in part explain our higher ${ }^{56} \mathrm{Ni}$ production. Another reason for the discrepancy is the different modeling approach of Fink et al. (2010). Their level set method does not allow a self-consistent calculation of the energy release and nuclear burning.

The abundances of the core detonation are in agreement with the qualitatively expected values. ${ }^{56} \mathrm{Ni}$ is produced most abundantly followed by ${ }^{28} \mathrm{Si}$ and ${ }^{32} \mathrm{~S}$. The mixing of carbon into the shell causes less carbon to be present in the core so that less is present after the detonation. The abundances of the core detonation are of the same order in Models M2a and FM3, except for the amount of ${ }^{12} \mathrm{C}$ which is about one order of magnitude lower in M2a than in FM3 after $100 \mathrm{~s}$ in our simulation. Small variations in the abundances of the core detonation between M2a and FM 3 can be explained by the different codes. The agreement of both models is better for the core detonation than the helium detonation as the core mass and composition are very similar. Moreover, the level set method to model detonations is more precise at the high densities in the core. The total final mass (shell and core) of ${ }^{56} \mathrm{Ni}\left(0.59 M_{\odot}\right)$ is in the expected range for a normal Type Ia supernova (Stritzinger et al. 2006; Scalzo et al. 2014). A discussion on how the change in the abundances influences the spectra follows in Section 5. 
$\mathrm{C} 12$

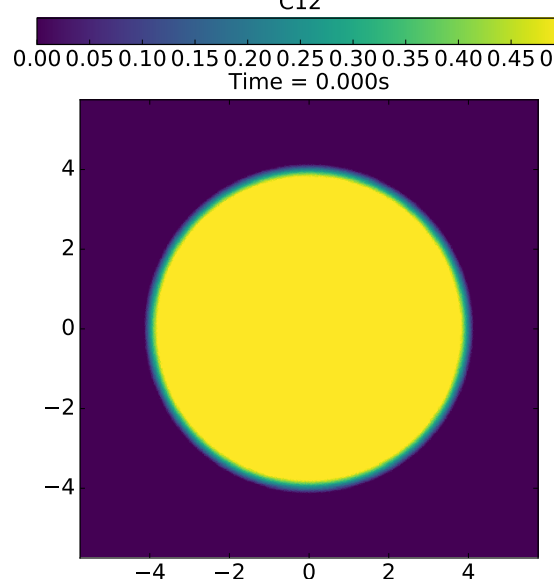

Time $=0.803 \mathrm{~s}$

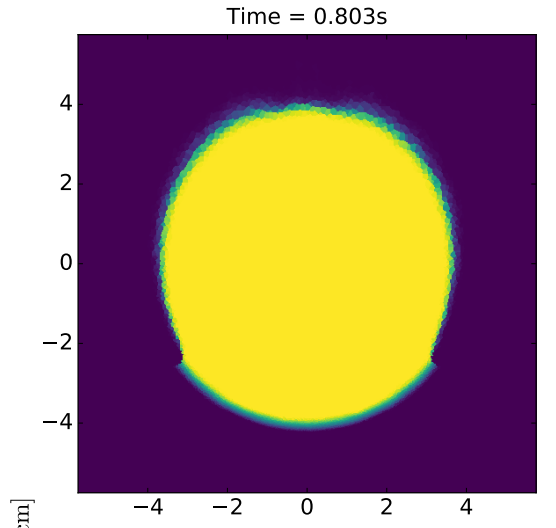

\begin{tabular}{l} 
్ㅓㅇ \\
$\infty$ \\
\\
\hdashline
\end{tabular}

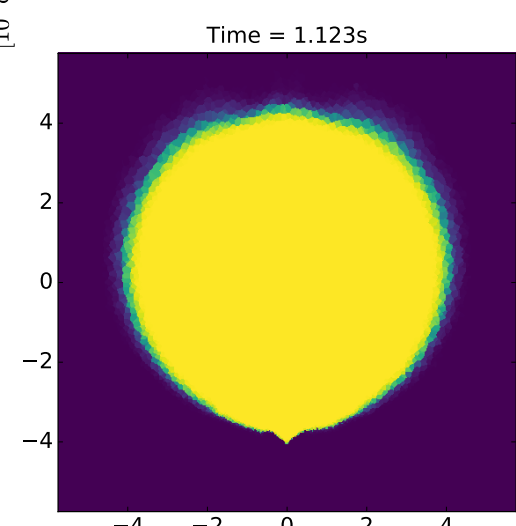

$\begin{array}{lllll}-4 & -2 & 0 & 2 & 4\end{array}$

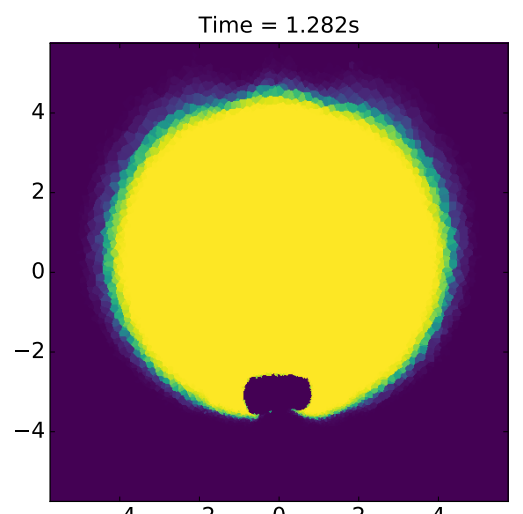

Temp $\left[10^{9} \mathrm{~K}\right]$
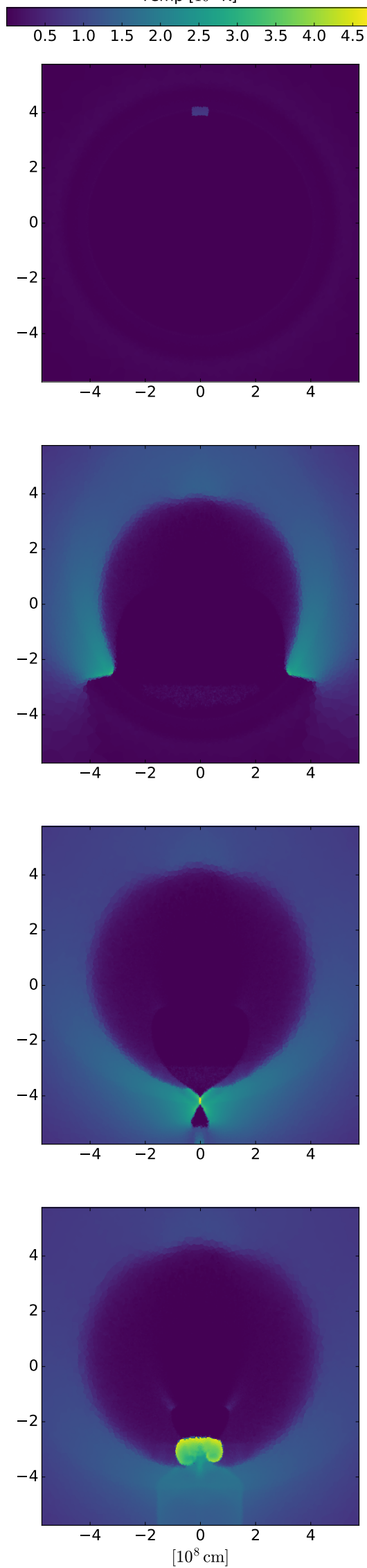

$\rho\left[\mathrm{g}\right.$ per $\left.\mathrm{cm}^{3}\right]$
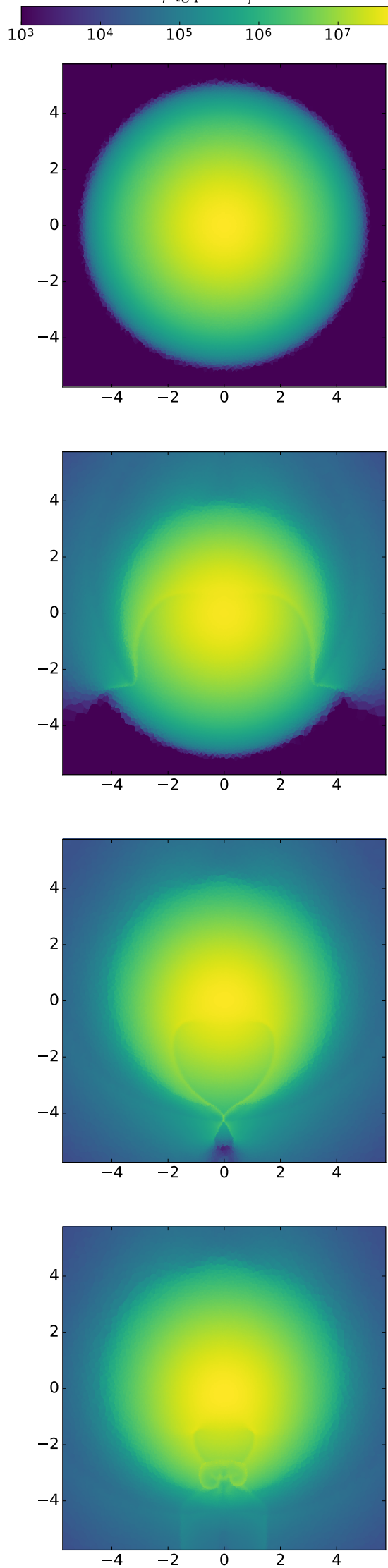

Fig. 3: Time evolution of Model M2a; shown from left to right: mass fraction of carbon, temperature in $\mathrm{K}$, and density in $\mathrm{g} \mathrm{cm}^{-3}$; shown from top to bottom: at time $t=0 \mathrm{~s}, t=0.803 \mathrm{~s}, t=1.123 \mathrm{~s}$, and $t=1.282 \mathrm{~s}$ plotted as slices through the center of the white dwarf in the $x-z$ plane. 

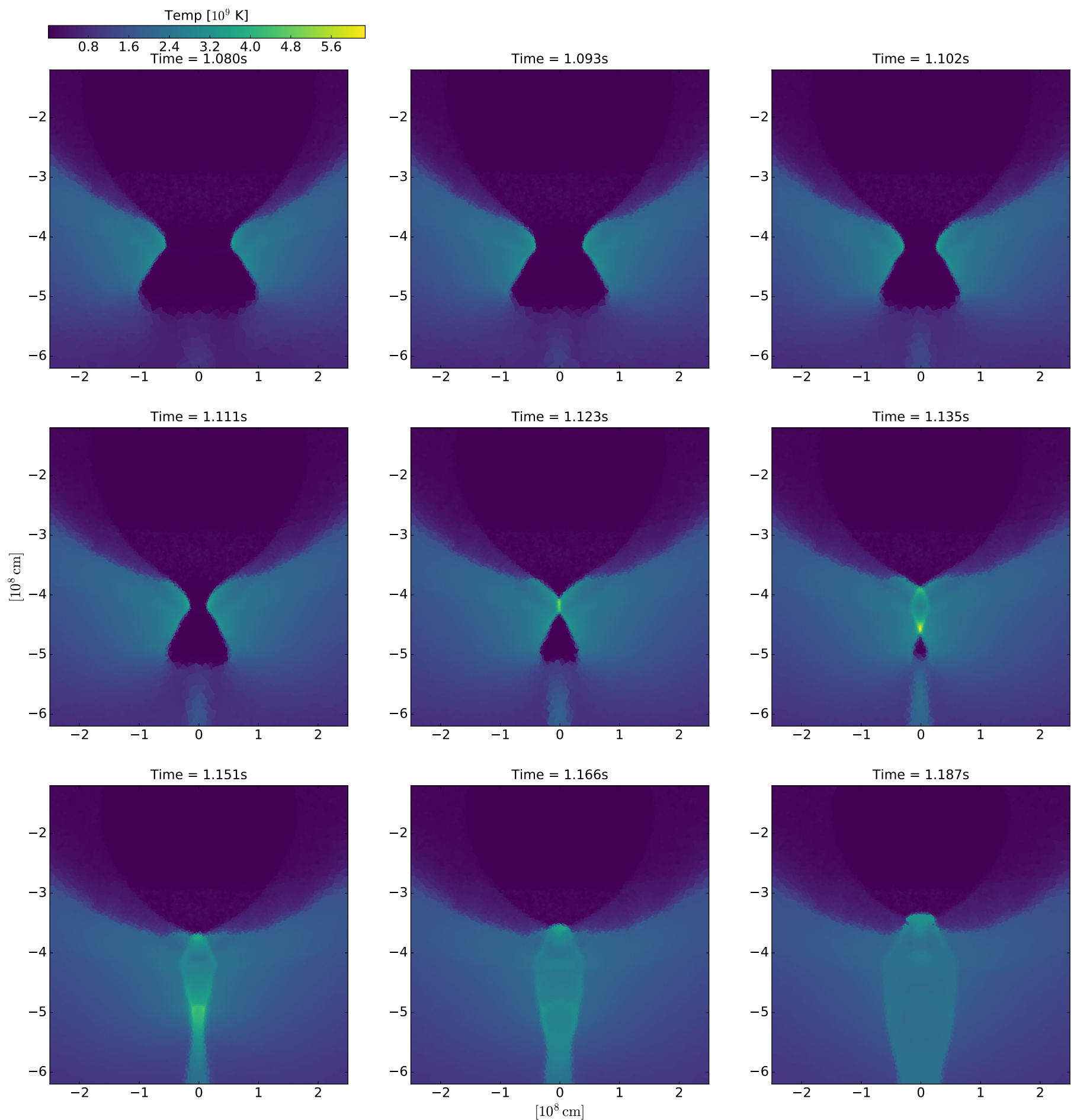

Fig. 4: Zoom-in of Fig. 3 on the time evolution of Model M2a; temperature profile with increasing time from left to right and top to bottom: the times are indicated above each plot going from $t=1.080 \mathrm{~s}$ in the top left to $t=1.187 \mathrm{~s}$ in the bottom right; plotted as slices through the center of the white dwarf in the $x-z$ plane.

\subsection{Influence of core and shell mixing}

Until now it is unclear whether the transition between core and shell is sharp or smeared out over a certain volume. Neunteufel et al. (2017) expect that some - but not very much mixing takes place during the accretion of helium onto the white dwarf. The ignition of He shell burning may dredge up some core material. Even without mixing, the material forming the $\mathrm{He}$ shell has some metallicity. Accretion from a hybrid HeCO WD is another possibility. An enrichment with carbon (and possibly other metals) influences the He burning. As a simple illustration, we consider the case of material consisting of ${ }^{4} \mathrm{He}$ and ${ }^{12} \mathrm{C}$ only. Similar arguments can be made for the admixture of other metals (see Shen \& Moore 2014). Carbon admixture in the He shell has two effects: it enhances the burning rate and can limit the mass number of the ash material. Kromer et al. (2010) show how this 
Table 2: Final abundances in the explosion ejecta for Models M1a, FM3 ${ }^{(1),(2)}$, M2a, and M2a_i55.

\begin{tabular}{lrrrr|rrrr}
\hline \multicolumn{9}{c}{ He detonation } \\
& M1a & FM3 & M2a & M2a_i55 & M1a & core detonation \\
& {$\left[\mathrm{M}_{\odot}\right]$} & {$\left[\mathrm{M}_{\odot}\right]$} & {$\left[\mathrm{M}_{\odot}\right]$} & {$\left[\mathrm{M}_{\odot}\right]$} & {$\left[\mathrm{M}_{\odot}\right]$} & {$\left[\mathrm{M}_{\odot}\right]$} & $\begin{array}{r}\text { M2a } \\
{\left[\mathrm{M}_{\odot}\right]}\end{array}$ & M2a_i55 \\
& {$\left[\mathrm{M}_{\odot}\right]$} \\
\hline${ }^{4} \mathrm{He}$ & $2.5 \times 10^{-2}$ & $3.3 \times 10^{-2}$ & $2.3 \times 10^{-2}$ & $2.3 \times 10^{-2}$ & $4.2 \times 10^{-3}$ & & $5.0 \times 10^{-3}$ & $5.4 \times 10^{-3}$ \\
${ }^{12} \mathrm{C}$ & $3.6 \times 10^{-4}$ & $2.2 \times 10^{-4}$ & $1.0 \times 10^{-4}$ & $6.8 \times 10^{-5}$ & $1.2 \times 10^{-3}$ & $2.7 \times 10^{-3}$ & $8.9 \times 10^{-4}$ & $8.2 \times 10^{-4}$ \\
${ }^{16} \mathrm{O}$ & $5.0 \times 10^{-3}$ & $1.9 \times 10^{-6}$ & $7.4 \times 10^{-3}$ & $7.6 \times 10^{-3}$ & $5.5 \times 10^{-2}$ & $8.0 \times 10^{-2}$ & $5.2 \times 10^{-2}$ & $5.2 \times 10^{-2}$ \\
${ }^{28} \mathrm{Si}$ & $4.6 \times 10^{-3}$ & $1.4 \times 10^{-4}$ & $8.9 \times 10^{-3}$ & $9.1 \times 10^{-3}$ & $1.7 \times 10^{-1}$ & $2.1 \times 10^{-1}$ & $1.6 \times 10^{-1}$ & $1.5 \times 10^{-1}$ \\
${ }^{32} \mathrm{~S}$ & $1.8 \times 10^{-3}$ & $7.8 \times 10^{-4}$ & $3.2 \times 10^{-3}$ & $3.3 \times 10^{-3}$ & $1.1 \times 10^{-1}$ & $1.0 \times 10^{-1}$ & $1.1 \times 10^{-1}$ & $1.0 \times 10^{-1}$ \\
${ }^{40} \mathrm{Ca}$ & $2.7 \times 10^{-3}$ & $2.2 \times 10^{-3}$ & $3.6 \times 10^{-3}$ & $3.5 \times 10^{-3}$ & $2.4 \times 10^{-2}$ & $1.8 \times 10^{-2}$ & $2.3 \times 10^{-2}$ & $2.2 \times 10^{-2}$ \\
${ }^{44} \mathrm{Ti}$ & $7.2 \times 10^{-4}$ & $3.4 \times 10^{-3}$ & $7.0 \times 10^{-4}$ & $6.9 \times 10^{-4}$ & $2.8 \times 10^{-5}$ & $1.1 \times 10^{-5}$ & $2.8 \times 10^{-5}$ & $2.9 \times 10^{-5}$ \\
${ }^{48} \mathrm{Cr}$ & $1.5 \times 10^{-3}$ & $4.4 \times 10^{-3}$ & $1.6 \times 10^{-3}$ & $1.6 \times 10^{-3}$ & $4.9 \times 10^{-4}$ & $4.5 \times 10^{-4}$ & $4.8 \times 10^{-4}$ & $4.7 \times 10^{-4}$ \\
${ }^{56} \mathrm{Ni}$ & $1.5 \times 10^{-2}$ & $1.7 \times 10^{-3}$ & $1.2 \times 10^{-2}$ & $1.2 \times 10^{-2}$ & $5.6 \times 10^{-1}$ & $5.5 \times 10^{-1}$ & $5.7 \times 10^{-1}$ & $5.9 \times 10^{-1}$ \\
\hline
\end{tabular}

References. (1) Fink et al. (2010), (2) Kromer et al. (2010)

impacts synthetic spectra and light curves and conclude that it leads to a better fit with the observations for Model FM3.

The triple- $\alpha$ reaction is the bottle-neck in the pure He composition case. In a pure He shell the triple- $\alpha$ reaction first needs to produce ${ }^{12} \mathrm{C}$ before the $\alpha$-process starting out with ${ }^{12} \mathrm{C}(\alpha, \gamma){ }^{16} \mathrm{O}$ forms heavier elements up to ${ }^{56} \mathrm{Ni}$. A seed abundance of carbon circumvents the triple- $\alpha$ bottleneck. This is because for the temperatures reached after ignition of explosive helium burning the $\alpha$-capture is faster than the triple- $\alpha$ reaction (see top panel of Fig. 5). Such an effect occurs for any carbon mass fraction above some cross-over temperature $T_{\mathrm{X}}$ (Fig. 5, bottom panel). Since $T_{\mathrm{X}}$ is typically smaller than the temperatures reached in explosive He burning, this enhances the burning rate in the $\mathrm{He}$ shell and leads to stronger shocks by increasing the energy release in the detonation.

The second effect in addition to this carbon-enhanced He detonation results from an over-pollution of the He shell with carbon: In the $\alpha$-process, it takes eleven $\alpha$ particles to reach ${ }^{56} \mathrm{Ni}$ from ${ }^{12} \mathrm{C}$. Therefore, for a number ratio of helium to carbon smaller than 11:1 we enter the $\alpha$-limited regime, where the $\alpha$ chain stalls around a nucleus with nucleon number $A<56$. The stagnation point of the $\alpha$-chain for a given carbon mass fraction is determined by the relations

$12+4 n=A$,

where $n$ is the number of $\alpha$ particles needed to reach the stagnation nucleus from ${ }^{12} \mathrm{C}$, and

$\frac{Y\left({ }^{4} \mathrm{He}\right)}{Y\left({ }^{12} \mathrm{C}\right)}=n=3 \frac{X\left({ }^{4} \mathrm{He}\right)}{X\left({ }^{12} \mathrm{C}\right)}$.

Since, by mass conservation of a two species mixture,

$X\left({ }^{4} \mathrm{He}\right)=1-X\left({ }^{12} \mathrm{C}\right)$,

Eq. (4) gives $n=3 / X\left({ }^{12} \mathrm{C}\right)-3$. Substituting $\mathrm{n}$ into Eq. (3) we finally get the nucleon number of the stagnation nucleus

$A=\frac{12}{X\left({ }^{12} \mathrm{C}\right)}$.

The $\alpha$-limited regime thus begins at a mass fraction $\mathrm{X}(\mathrm{C})>0.21$. In their Model 3m, Kromer et al. (2010) add 34\% by mass of ${ }^{12} \mathrm{C}$ to the He shell and therefore reach a stagnation of the $\alpha$-process around argon which avoids strong imprints on the predicted optical spectra of the simulated supernova explosion. While Kromer et al. (2010) study a homogeneous admixture of carbon to the shell, our models are characterized by an abundance profile with a higher amount of carbon at the base of the shell than at its outer edge that results from the relaxation process (see Section 3.2). This gradient represents the expected abundance distribution more realistically (Neunteufel et al. 2017).

To bracket the effect and to investigate the influence of carbon in the shell on the detonation ignition mechanism, a different structure in the helium, carbon, and oxygen abundances is modeled. For this, no additional mixing of carbon and oxygen into the He shell is added after the relaxation in Model M1a: In this model the core consists of pure carbon and oxygen in equal mass parts and the helium shell is only slightly enriched with carbon. As a result of this only about $0.007 M_{\odot}$ of each carbon and oxygen is present in the shell and the total shell mass decreases. The change in the composition of the transition region has an influence on the carbon detonation ignition mechanism. Contrary to Model M2a, the convergence of the helium detonation wave at the antipode is not strong enough to ignite a carbon detonation at the shell-core interface in Model M1a. However, a detonation according to the converging shock mechanism is found. Moreover, the omission of additional mixing in Model M1a results in small differences of the final abundances compared to those of Model M2a. Because of the similar shell mass and detonation ignition mechanism the abundances of Model M1a can best be compared to Model FM3 (see Table 2). The small addition of carbon to the shell in the transition region leads to an increased production of IMEs compared to FM3 while one order of magnitude less ${ }^{44} \mathrm{Ti}$ and more ${ }^{56} \mathrm{Ni}$ is produced in the shell detonation. These differences can be explained by the different treatment of the helium shell detonation in both models.

Models M1a and 3m by Kromer et al. (2010) lead us to conclude that the mixing of carbon to the helium shell has an impact on the yields as it results in the production of heavier elements. However, our models show that it does not solve the problem of a significant amount of ${ }^{4} \mathrm{He}$ being unburned and the redness of the synthetic spectra (Boyle et al. 2017; Botyánszki et al. 2018). On the other hand the mixing is critical for the details of the detonation ignition mechanism as stated above.

\subsection{Resolution study}

In this study, we are interested in the results of the helium shell detonation and the mechanism of the core detonation ignition. 
Table 3: Reference mass $M_{\mathrm{R}}$ of the helium shell and at the carbon ignition point as well as energy release of the He shell detonation of Models M2a, M2a_13, M2a_21, M2a_36, and M2a_79. The last column lists whether a carbon detonation ignition following the scissors mechanism is observed.

\begin{tabular}{lrrrr}
\hline & $\begin{array}{r}M_{\mathrm{R}} \text { in He shell } \\
{\left[10^{26} \mathrm{~g}\right]}\end{array}$ & $\begin{array}{r}\mathrm{E}_{\mathrm{He} \text { shell }}[\mathrm{erg}] \\
\text { M2a_79 }\end{array} 20.0$ & $\begin{array}{r}M_{\mathrm{R}} \text { at C ign. point } \\
{\left[10^{26} \mathrm{~g}\right]}\end{array}$ & scissors mechanism \\
M2a_36 & 4.0 & $9.93 \times 10^{49}$ & & \\
M2a_21 & 2.0 & $9.97 \times 10^{49}$ & 2.0 & no \\
M2a_13 & 2.0 & & 1.2 & yes \\
M2a & 2.0 & & 0.2 & yes \\
\hline
\end{tabular}
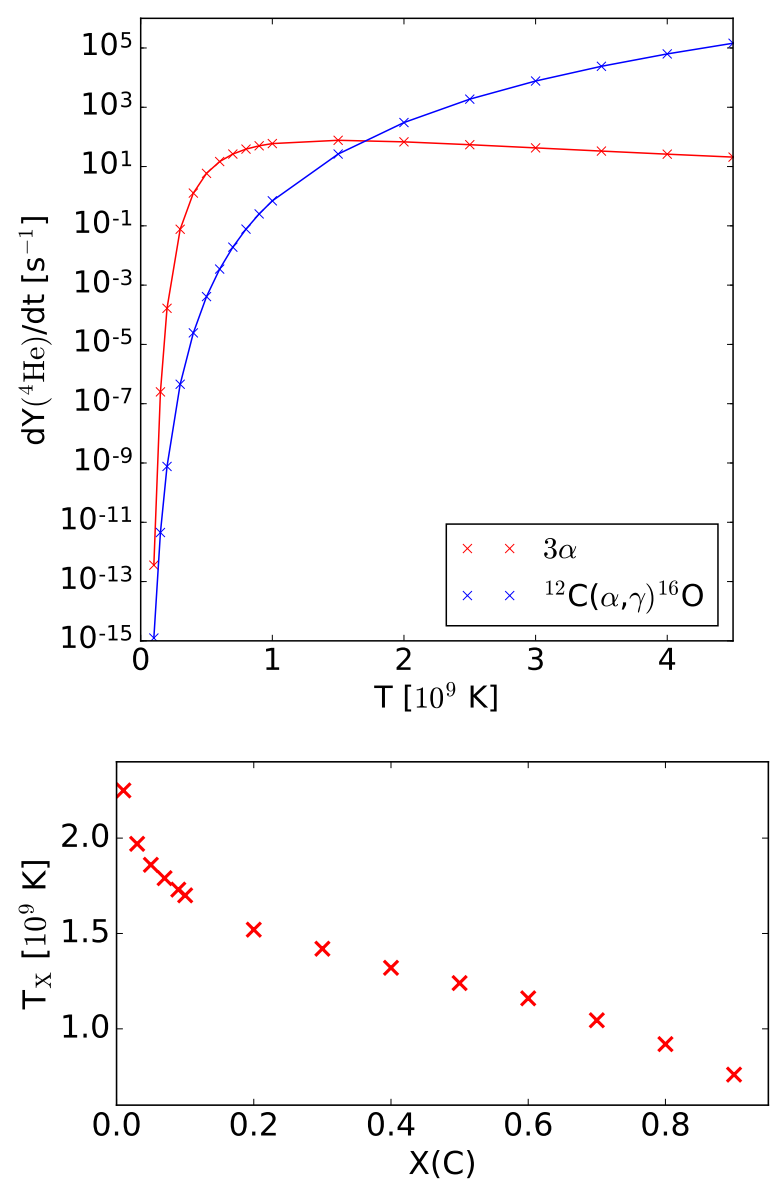

Fig. 5: Top: Rate of change in ${ }^{4} \mathrm{He}$ abundance due to the triple- $\alpha$ (red) and ${ }^{12} \mathrm{C}(\alpha, \gamma){ }^{16} \mathrm{O}$ (blue) reactions at a density of $1.2 \times 10^{6} \mathrm{~g} \mathrm{~cm}^{-3}$ and $X\left({ }^{12} \mathrm{C}\right)=0.1$ dependent on temperature; bottom: crossing temperature $T_{\mathrm{X}}$ above which the depletion due to $\alpha$-captures exceeds that due to triple- $\alpha$ dependent on initial carbon abundance at a density of $1.2 \times 10^{6} \mathrm{~g} \mathrm{~cm}^{-3}$. The reaction rates are taken from the JINA Reaclib Database (Cyburt et al. 2010) based on Xu et al. (2013) for the $\alpha$-capture and Fynbo et al. (2005) for the triple- $\alpha$ reaction.

We therefore perform numerical convergence studies for the two effects in separate steps. Models M2a_79, M2a_36, and M2a_21 have different refinements in the helium shell, characterized by the reference mass $M_{\mathrm{R}}$ as given in the first column of Tab. 3. The total number of cells $1.123 \mathrm{~s}$ after helium ignition is 2.0 million, 1.6 million, and 1.3 million for Models M2a_21, M2a_36, and M2a_79. The energy release of the He shell detonation (see sec- ond column of Tab. 3) shows convergence: the difference of the energy release between the simulation with the high (M2a_21) and modest (M2a_36) resolution is smaller than the difference between M2a_36 and M2a_79.

Model M2a_21, which has the highest He shell refinement, is taken as the base for testing the convergence of the carbon detonation ignition mechanism. Two further simulations have an additional refinement around the carbon ignition point (see third column in Tab. 3). A detonation ignition by the scissors mechanism is not observed in Model M2a_21, but for both models with higher resolution in the corresponding material. This indicates that core detonation ignition by the scissors mechanism is the converged numerical solution.

\subsection{Sensitivity to the ignition spot}

As described in Section 3.3 the ignition spot for the first detonation was changed to consider two different locations in Model M2. The simulations M2a and M2b differ only slightly (see Table 1). The same propagation behavior is observed when the ignition spot is set to be located at the base of the He shell (Model $\mathrm{M} 2 \mathrm{~b}$ ) and a carbon detonation is ignited at the same location as in Model M2a. The detonation ignition mechanism is robust against small changes in the location of the first ignition spot of the helium detonation.

\subsection{Influence of a different white dwarf mass}

Table 4: Final abundances for Model M3a and FM1 $1^{(1),(2)}$.

\begin{tabular}{|c|c|c|c|c|}
\hline & \multicolumn{2}{|c|}{ He detonation } & \multicolumn{2}{|c|}{ core detonation } \\
\hline & M3a & FM1 & M3a & FM1 \\
\hline & {$\left[\mathrm{M}_{\odot}\right]$} & {$\left[\mathrm{M}_{\odot}\right]$} & {$\left[\mathrm{M}_{\odot}\right]$} & {$\left[\mathrm{M}_{\odot}\right]$} \\
\hline${ }^{4} \mathrm{He}$ & $4.2 \times 10^{-2}$ & $8.3 \times 10^{-2}$ & $1.4 \times 10^{-3}$ & \\
\hline${ }^{12} \mathrm{C}$ & $7.6 \times 10^{-5}$ & $1.2 \times 10^{-3}$ & $4.0 \times 10^{-4}$ & $6.6 \times 10^{-3}$ \\
\hline${ }^{16} \mathrm{O}$ & $1.7 \times 10^{-2}$ & $3.2 \times 10^{-6}$ & $6.8 \times 10^{-2}$ & $1.4 \times 10^{-1}$ \\
\hline${ }^{28} \mathrm{Si}$ & $2.7 \times 10^{-2}$ & $4.8 \times 10^{-4}$ & $1.8 \times 10^{-1}$ & $2.7 \times 10^{-1}$ \\
\hline${ }^{32} \mathrm{~S}$ & $5.0 \times 10^{-3}$ & $2.2 \times 10^{-3}$ & $1.2 \times 10^{-1}$ & $1.3 \times 10^{-1}$ \\
\hline${ }^{40} \mathrm{Ca}$ & $4.2 \times 10^{-3}$ & $4.7 \times 10^{-3}$ & $2.3 \times 10^{-2}$ & $2.0 \times 10^{-2}$ \\
\hline${ }^{44} \mathrm{Ti}$ & $1.3 \times 10^{-3}$ & $7.9 \times 10^{-3}$ & $1.9 \times 10^{-5}$ & $7.2 \times 10^{-6}$ \\
\hline${ }^{48} \mathrm{Cr}$ & $2.5 \times 10^{-3}$ & $1.1 \times 10^{-2}$ & $4.4 \times 10^{-4}$ & $3.9 \times 10^{-4}$ \\
\hline${ }^{56} \mathrm{Ni}$ & $3.1 \times 10^{-2}$ & $8.4 \times 10^{-4}$ & $3.1 \times 10^{-1}$ & $1.7 \times 10^{-1}$ \\
\hline
\end{tabular}

References. (1) Fink et al. (2010), (2) Kromer et al. (2010) 
A comparison of Model M2a to Model M3a is carried out to consider the effects different white dwarf masses might have on the mechanism. Details of Model M3a are listed in Table 1. The size of the helium detonation is slightly smaller than in Model M2a. 2447 cells are ignited with $\Delta R$ set to be 0.02 times the distance between central detonating cell and the center of the white dwarf as described in Sec. 3.3. The total mass of the white dwarf here is about $0.9 \mathrm{M}_{\odot}$ with a helium shell about twice as massive as in Model M2a. The simulation is run at a high resolution and shows a convergence of the detonation wave $1.251 \mathrm{~s}$ after the first ignition. At this point, opposite to the helium detonation spot, a density of at least $5.8 \times 10^{6} \mathrm{~g} \mathrm{~cm}^{-3}$ and temperature higher than $3.2 \times 10^{9} \mathrm{~K}$ is reached in cells with a mass fraction of 0.32 in ${ }^{12} \mathrm{C}$ leading to the same detonation ignition mechanism as in Model M2a.

The final abundances gained from Model M3a are listed in Table 4. A total of $0.34 \mathrm{M}_{\odot}$ of ${ }^{56} \mathrm{Ni}$ is produced in the shell and core detonations combined. The total yields for ${ }^{28} \mathrm{Si}$ and ${ }^{32} \mathrm{~S}$ are produced most abundantly following ${ }^{56} \mathrm{Ni}$ in this simulation with $0.21 \mathrm{M}_{\odot}$ and $0.12 \mathrm{M}_{\odot}$, respectively. The final abundances of Model FM1 are included in Table 4 for comparison. Due to the relaxation process and mixing of carbon and oxygen into the shell in our simulation the He shell is about $0.03 \mathrm{M}_{\odot}$ heavier than in Model FM1. This leads to a much higher production of ${ }^{56} \mathrm{Ni}$ and IMEs during the He detonation, but lower yields of ${ }^{48} \mathrm{Cr}$. The abundances from the core detonation show the same relations as Models M2a and FM3 for most isotopes. Only the differences in the ${ }^{16} \mathrm{O}$ and ${ }^{56} \mathrm{Ni}$ abundances are higher as Model M3a burns more oxygen and produces twice as much nickel as the model by Fink et al. (2010). We remind the reader that Fink et al. (2010) uses a level set method which is different from our numerical treatment. Their approach is better suited for a simulation of the WD core at high densities than that of the helium shell detonation, as discussed in Sec. 4.2.

\subsection{Influence of the nuclear network}

We test the sensitivity of the results to the size of the employed nuclear network. For this we consider a 55-isotope nuclear network during the hydrodynamics simulation. It is chosen to match the nuclear network in Townsley et al. (2019) consisting of $\mathrm{n}, \mathrm{p},{ }^{4} \mathrm{He},{ }^{11} \mathrm{~B},{ }^{12-13} \mathrm{C},{ }^{13-15} \mathrm{~N},{ }^{15-17} \mathrm{O},{ }^{18} \mathrm{~F},{ }^{19-22} \mathrm{Ne},{ }^{22-23} \mathrm{Na}$, ${ }^{23-26} \mathrm{Mg},{ }^{25-27} \mathrm{Al},{ }^{28-30} \mathrm{Si},{ }^{29-31} \mathrm{P},{ }^{31-33} \mathrm{~S},{ }^{33-35} \mathrm{Cl},{ }^{36-39} \mathrm{Ar},{ }^{39} \mathrm{~K}$, ${ }^{40} \mathrm{Ca},{ }^{43} \mathrm{Sc},{ }^{44} \mathrm{Ti},{ }^{47} \mathrm{~V},{ }^{48} \mathrm{Cr},{ }^{51} \mathrm{Mn},{ }^{52,56} \mathrm{Fe},{ }^{55} \mathrm{Co}$, and ${ }^{56,58-59} \mathrm{Ni}$. Shen \& Moore (2014) point out that a large nuclear network is needed to model the nuclear energy release accurately. Following this, Townsley et al. (2019) show that a 55 isotope nuclear network is large enough. They argue that their isotope network gives the same energy release as a large 495-isotope network. The nuclear network of Townsley et al. (2019) best captures He burning at low densities while the 33 isotope nuclear network used in M2a is optimized to follow carbon burning. As our models do not consider a helium shell enriched with ${ }^{14} \mathrm{~N}$, there is no need to include a larger nuclear network similar to the one by Townsley et al. (2019).

In our Model M2a_i55, considering the 55 isotope nuclear network, the second detonation is ignited in the same way as in Model M2a though the ignition occurs $0.003 \mathrm{~s}$ later. We further find that the total energy release of the shell and core detonation are within a few per cent of each other for Models M2a and M2a_i55 (see Tab. 5). The final abundances are included in Table 2 and it is shown that the different nuclear network does not change the abundances significantly.
Table 5: Energy release of Models M2a and M2a_i55.

\begin{tabular}{lrr}
\hline & $\begin{array}{r}\text { He detonation } \\
{[\mathrm{erg}]}\end{array}$ & $\begin{array}{r}\text { core detonation } \\
{[\mathrm{erg}]}\end{array}$ \\
\hline M2a & $9.93 \times 10^{49}$ & $1.35 \times 10^{51}$ \\
M2a_i55 & $1.01 \times 10^{50}$ & $1.34 \times 10^{51}$ \\
\hline
\end{tabular}

\subsection{Comparison to previous work}

Livne \& Arnett (1995), García-Senz et al. (1999), Forcada et al. (2006), and Forcada (2008) found a similar carbon detonation ignition mechanism in their models. Livne \& Arnett (1995) and García-Senz et al. (1999) consider different masses in their work. García-Senz et al. (1999) simulate the explosion of a white dwarf with a total mass of $1.02 \mathrm{M}_{\odot}$ and Livne \& Arnett (1995) look into different total masses between $0.70 \mathrm{M}_{\odot}$ and $1.10 \mathrm{M}_{\odot}$. Differences in the setup, namely the core and shell masses, do not allow us to compare the final abundances of the models with our work. The model of Forcada et al. (2006) and Forcada (2008) has a total mass of $0.9 \mathrm{M}_{\odot}$. Their helium shell is more massive $\left(0.2 \mathrm{M}_{\odot}\right)$ than our Model M3a which makes a comparison difficult. Simulations by García-Senz et al. (2018) show the same detonation ignition mechanism. However, they consider different masses for their models and look into the effect of rotation on the detonation mechanism.

The different models confirm that the detonation ignition mechanism is not limited to one specific setup as shown in Section 4.6. Forcada (2008) finds that the location of the He ignition is important for the success of the mechanism. Three of their models show a direct carbon detonation ignition at the surface of the core matching the edge-lit mechanism. The success of the scissors mechanism may also depend on the thickness of the transition region between core and shell. Our simulations do not show a prompt edge-lit detonation (as for example Forcada 2008). García-Senz et al. (2018) find that the convergence of the He detonation waves weakens under the effect of rotation. Nevertheless, an ignition of the core is still observed at a later point.

Unlike Livne \& Arnett (1995), García-Senz et al. (1999), Forcada (2008), and García-Senz et al. (2018), work by for instance Fink et al. (2007) does not investigate the possibility of a mechanism where the convergence of the detonation wave in the helium shell leads to a second detonation. This is also due to the fact that they use the level set method to track detonations which prevents an automatic ignition of the second detonation, but the core detonation has to be ignited by hand. They therefore state that their results confirm that a core detonation would ignite in the converging shock mechanism in case a detonation is not triggered already at the edge of the core.

\section{Synthetic observables}

To explore the observable consequences of the scissors mechanism, we have performed radiative transfer calculations for our reference model M2a. We selected this model for particular study since it has brightness appropriate for a typical Type Ia supernova and can be closely compared to results from the converging shock double-detonation Model FM3.

We have also computed synthetic observables for Models M1a and M2a_i55. These allow us to quantify the effect of mixing in Model M2a compared to Model M1a, and also the effect of using a 33 isotope nuclear network, compared to Model M2a_i55 which used a 55 isotope nuclear network. We reserve 
for future studies a broader, systematic study of a range of combined $\mathrm{CO}$ and He progenitor masses (models such as M3a). We recalculated the synthetic observables for Model FM3 in order to ensure that any differences are due to the explosion models, and not differences in the setup of our radiative transfer calculations.

In the following we first compare the angle-averaged light curves and spectra between the models, and comment on their comparison to observations. We then discuss the viewing angle effects for our synthetic observables.

\subsection{Angle averaged light curves}

The model angle averaged light curves are shown in Fig. 6, and compared to Model FM3. The parameters for these light curves are summarized in Table 6.

Table 6: Observable parameters of Models M2a, FM3, M1a, and M2a_i55.

\begin{tabular}{lrrrr}
\hline & M2a & FM3 & M1a & M2a_i55 \\
\hline$\Delta \mathrm{m}_{15}(\mathrm{~B})(\mathrm{mag})$ & 1.82 & 2.00 & 1.62 & 1.83 \\
$\mathrm{t}_{\max }(\mathrm{B})(\mathrm{d})$ & 16.6 & 17.7 & 16.4 & 16.6 \\
$\mathrm{M}_{\mathrm{U}, \max }(\mathrm{mag})$ & -18.7 & -18.4 & -18.5 & -18.7 \\
$\mathrm{M}_{\mathrm{B}, \max }$ (mag) & -18.9 & -18.6 & -18.7 & -18.9 \\
$\mathrm{M}_{\mathrm{V}, \max }(\mathrm{mag})$ & -19.8 & -19.7 & -19.8 & -19.8 \\
$\mathrm{M}_{\mathrm{R}, \max }(\mathrm{mag})$ & -19.6 & -19.6 & -19.6 & -19.6 \\
$\mathrm{M}_{\mathrm{I}, \max }(\mathrm{mag})$ & -19.2 & -19.2 & -19.2 & -19.2 \\
$(\mathrm{U}-\mathrm{B})_{\mathrm{B}, \max }(\mathrm{mag})$ & 0.24 & 0.24 & 0.36 & 0.26 \\
$(\mathrm{~B}-\mathrm{V})_{\mathrm{B}, \max }(\mathrm{mag})$ & 0.81 & 0.95 & 0.92 & 0.82 \\
$(\mathrm{~V}-\mathrm{R})_{\mathrm{B}, \max }(\mathrm{mag})$ & -0.099 & -0.016 & -0.099 & -0.093 \\
$(\mathrm{~V}-\mathrm{I})_{\mathrm{B}, \max }(\mathrm{mag})$ & -0.44 & -0.39 & -0.42 & -0.47 \\
\hline
\end{tabular}

Model M2a was chosen to be similar in mass to Model FM3, allowing a close comparison between the outcomes of the explosion mechanisms for similar progenitor configurations. The shapes of the light curves are similar for these two models, despite the difference in the detonation mechanism (the scissors mechanism for M2a and converging shock for FM3). However, in the B band M2a is 0.3 mag brighter than FM3, peaks $\sim 1$ day earlier, and declines more slowly from maximum. These effects can likely be attributed to the higher abundance of ${ }^{56} \mathrm{Ni}$ synthesized in the helium detonation.

Using a 55 isotope nuclear network, as discussed in Section 4.2, rather than our standard 33 isotope network, makes only slight differences to the light curves of Model M2a_i55 compared to Model M2a. Both models have the same peak brightnesses in the bands shown in Fig. 6, and show very similar declines from maximum over 15 days in the B band. Given the similarities of these models we conclude that using a 55 isotope network during the hydrodynamics simulation does not have a significant effect on the model light curves.

As described in Section 4.3, Model M1a investigates the influence of core and shell mixing on the detonation ignition mechanism. In Model M1a the abundances are reset after relaxation to bracket the effect of mixing in M2a. This resulted in not achieving a detonation by the scissors mechanism, as for Model M2a, but by the converging shock mechanism. M1a peaks 0.2 days before Model M2a, is 0.2 mag fainter at maximum in the $\mathrm{U}$ and B bands, and the same in the V, R, and I bands. The differences in these explosion models make subtle changes to the light curves, however, these differences are small, for example in comparison to the scale of discrepancies with data (see Section 5.4).

The color evolution for these models is shown in Fig. 7. At times before maximum, the B-V colors of the models exploded by the scissors mechanism (Model M2a and Model M2a_i55) are marginally bluer than those exploded by the converging shock mechanism (Model M1a and Model FM3). At later times both the B-V colors and the V-R colors are redder for Model FM3 than for Models M2a, M1a, and M2a_i55. Our models all show a very similar color evolution. The greatest differences seen are for Model FM3 in comparison to the new models, however, these are still small and do not dramatically affect the level of agreement to be found in comparison to data.

\subsection{Angle averaged spectra}

In order to understand the elemental contributions responsible for shaping the spectra, we indicate the spectral contributions to emission and absorption, identified by ion for Model M2a at 18 days after explosion in Fig. 8. Specifically, in the Monte Carlo simulations, we record details of the last interaction each escaping Monte Carlo packet underwent. For each wavelength bin in the synthetic spectrum we then color code the area under the spectrum in proportion to the energy carried by packets in that bin whose last interaction was with each of the ions considered. We also construct an equivalent histogram of color coded contributions based on where the wavelength bin packets were prior to their last interaction (i.e. indicating where packets last underwent absorption/scattering/fluorescence) and plot this under the spectrum as an indication of the key absorption processes. This analysis confirms that the helium shell ash causes strong absorption features in the bluer regions of the spectrum, as has been previously shown (Kromer et al. 2010).

The spectra for the models are compared in Fig. 9 at 10 days and 18 days after explosion. As expected from the similarities between the light curves for these models, the spectra for each of the models do not show significant differences. Again we see that the most prominent differences are for Model FM3. At 10 days the $\mathrm{Si}$ II emission at $\approx 6400 \AA$ and Ca II emission at $\approx 8500 \AA$ is weaker for Model FM3, and the strength of Ti II absorption, especially at 10 days, is strongest for Model FM3.

\subsection{Comparison objects}

We compare our models to SN 2011fe (Nugent et al. 2011), SN 2016jhr (Jiang et al. 2017), and SN 2018byg (De et al. 2019). Out of these, SN 2011fe is a very well-observed Type Ia supernova of normal brightness, and is spectroscopically normal. It is therefore a suitable benchmark to judge the validity of our models for rather normal SNe Ia.

SN 2016jhr was specifically suggested to have been triggered by a helium shell detonation. It showed a prominent early optical flash $\sim 0.5$ days after explosion, an early red and rapid color evolution, and a light curve typical of normal brightness Type Ia supernovae, but showed strong titanium absorption, which is typically seen in the spectra of sub-luminous supernovae. Jiang et al. (2017) attribute the early flash to the decays of ${ }^{56} \mathrm{Ni}$ and other radioactive isotopes in the outer layers of the ejecta, produced in the helium detonation. They ruled out interaction between the ejecta and either circumstellar material or a companion star as the cause of the early flash due to the early red color, as their models showed a bluer color evolution for these scenarios. 


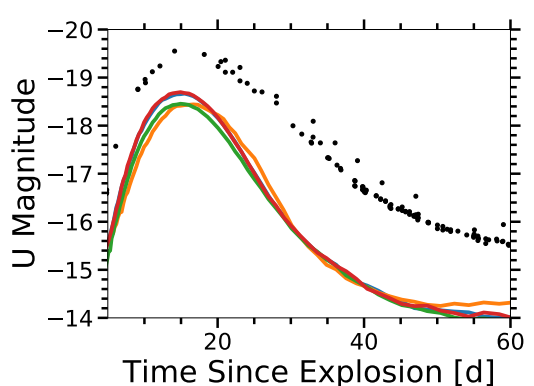

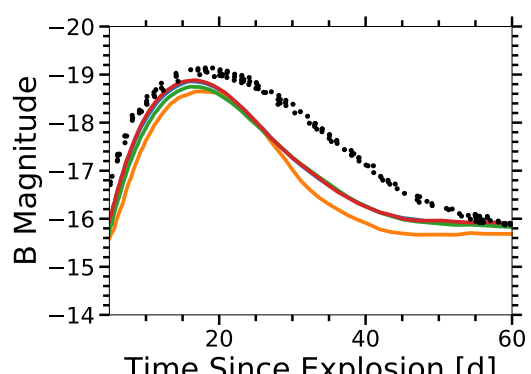

Time Since Explosion [d]

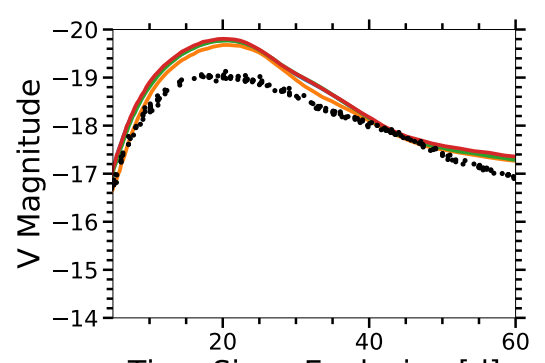

Time Since Explosion [d]
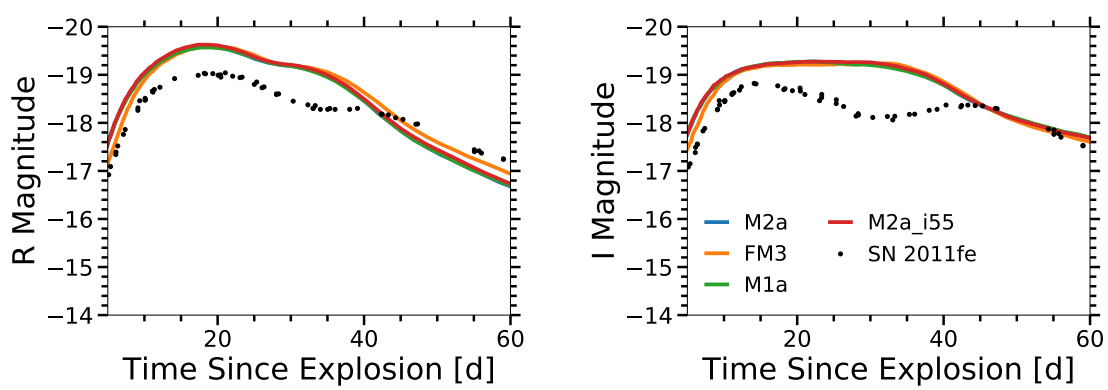

Fig. 6: Angle averaged U, B, V, R, and I band limited light curves for our models as well as Model FM3 compared to the spectroscopically normal Type Ia supernova SN 2011fe (Nugent et al. 2011).
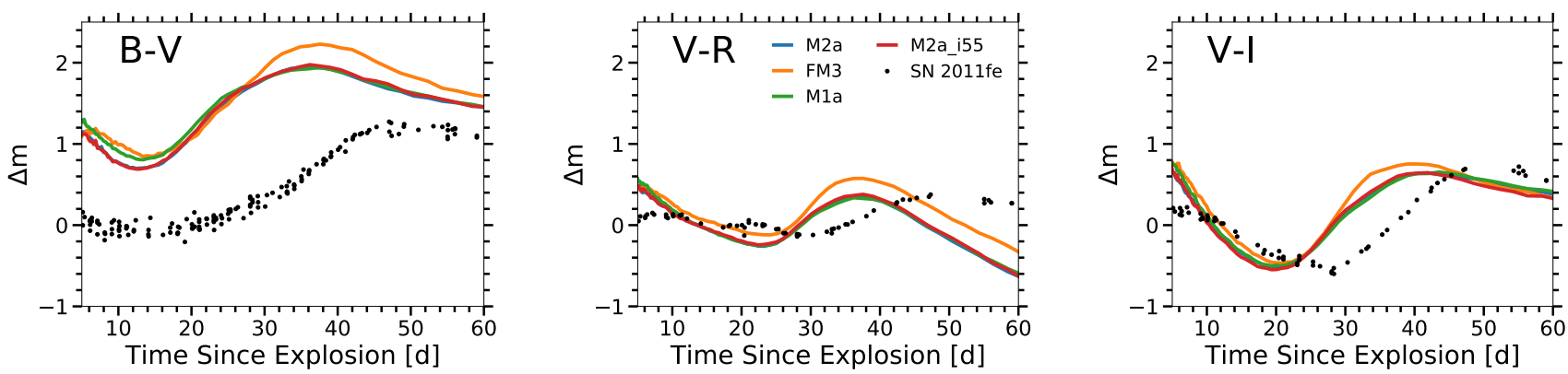

Fig. 7: Angle averaged B-V, V-R, and V-I color curves of the same models as in Fig. 6. For comparison we plot the colors of the spectroscopically normal SN 2011fe (Nugent et al. 2011).

Both the objects mentioned above (SN 2011fe and 2016jhr) are of similar peak brightness to our models. We also make comparisons of our models with SN 2018byg, which - although significantly fainter - has also been suggested to be the result of a helium-shell double detonation on a sub-Chandrasekhar-mass white dwarf. The bluer regions of the spectra of SN 2018byg show unusually strong line blanketing, with broad Ti II and Fegroup element absorption features, and near peak they observe a deep, high velocity $\left(\approx 25000 \mathrm{~km} \mathrm{~s}^{-1}\right.$ ) Ca II triplet absorption feature. The light curves of SN 2018byg are sub-luminous and similar to SN 1991bg-like Type Ia supernovae, except for a rapid rise in r-band magnitude within the first week from explosion. Our models are therefore systematically too bright compared to SN 2018byg, however, we include this to make spectral comparisons due to the similar nature of the proposed explosion scenario. An explosion driven by a helium detonation naturally explains the high velocity $\mathrm{Ca}$ II feature, as a helium detonation produces calcium, and other intermediate mass elements, in the outer layers of the ejecta. De et al. (2019) find that the early, fast rise in the r-band light curve is consistent with the presence of radioactive material in the outer ejecta from a helium shell detonation.

\subsection{Comparison to observations}

To compare our models with observations we first only discuss the angle averaged light curves and spectra for our models. We discuss viewing angle effects in Section 5.5. We compare the model light curves to our comparison objects, followed by a comparison of the model colors to these objects. The spectra are discussed afterwards. We correct the spectra and photometry for SN 2016jhr and SN 2018byg for reddening due to Galactic extinction (given by $\mathrm{E}(\mathrm{B}-\mathrm{V})_{\mathrm{MW}}=0.0263 \mathrm{mag}$ (Jiang et al. 2017) and $\mathrm{AV}=0.032 \mathrm{mag}$ (De et al. 2019), respectively). The total extinction to SN $2011 \mathrm{fe}$ was found to be negligible (Nugent et al. 2011). The spectra of all our comparison objects are redshift corrected.

The U, B, V, R, and I band light curves of SN 2011fe are included in Fig. 6. We find that our models are of similar brightness in the B band until around maximum. As discussed by Kromer et al. (2010) for Model FM3, our models decline from maximum 


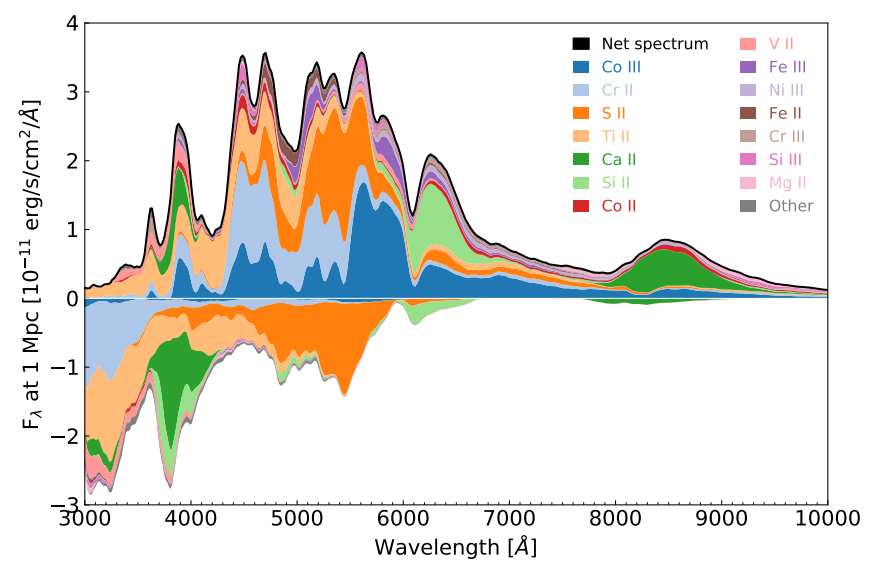

Fig. 8: Angle averaged emission and absorption spectrum for Model M2a at 18 days after explosion. The total emission spectrum is plotted in black. The color coding indicates the ions responsible for the emission and absorption. The ions are listed in the legend of this figure in order of greatest contribution of flux.
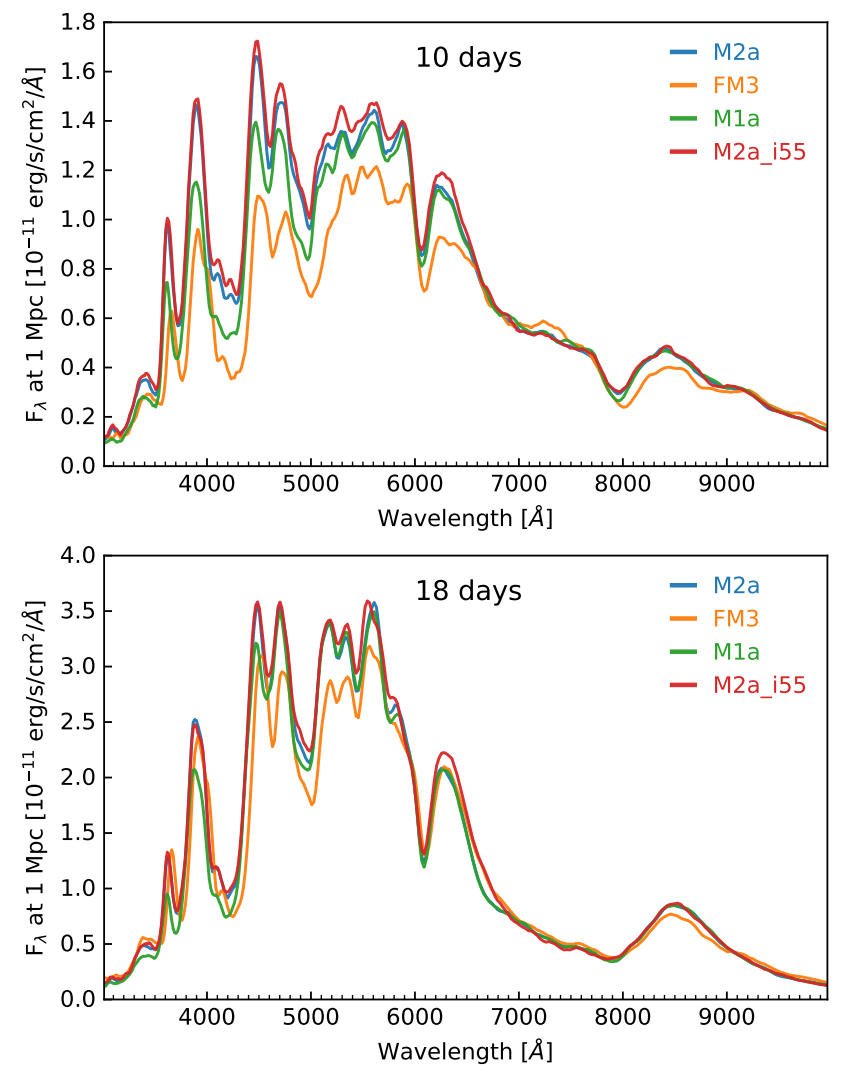

Fig. 9: Comparison of the spectra for Models M2a, FM3, M1a, and M2a_i55 at 10 days (upper panel) and 18 days (lower panel) after maximum light.

in the B band too rapidly compared to observed normal SNe Ia of comparable brightness. We also find our models to be too faint in the $\mathrm{U}$ band. This region of the spectrum is strongly affected by absorption from the helium shell ash. In the V, R, and I bands we find that our models are brighter than SN 2011fe around peak, but are of similar brightness at later phases.

The B band maximum of SN 2016jhr is -18.8 mag (derived by Jiang et al. 2017), which is similar to our reference model M2a, however, SN 2016jhr peaks at $\sim 20$ days after explosion while Model M2a peaks at 16.6 days after explosion. Again, our models decline more rapidly in the B band than SN 2016jhr, which has a $\Delta \mathrm{m}_{15}(\mathrm{~B})$ of $\sim 1 \mathrm{mag}$.

SN 2016jhr has a redshift of $z=0.11737$, and SN 2018byg has a redshift of $\mathrm{z}=0.066304$. Hence the effects on the light curves due to redshift are not negligible. To show the extent of this effect, and to make a direct comparison to SN 2016jhr, we plot the light curves of Model M2a from our synthetic spectra after they have been redshifted to $\mathrm{z}=0.11737$ in Fig. 10 . We also account for the time dilation at this redshift. The $g$, r, and i band light curves of SN 2016jhr and SN 2018byg are plotted, however, we note that this is not a direct comparison for SN 2018byg given the lower redshift of this object. Model M2a declines more rapidly from maximum in the $\mathrm{g}$ band than SN 2016jhr, and is brighter in the $r$ band near maximum. As SN 2018byg is sub-luminous, the brightnesses of our model light curves do not match this object. Future work will include a parameter study that investigates models of lower luminosity.

The angle-averaged time evolution of the B-V, V-R, and VI colors are shown in Fig. 7, and compared to SN 2011fe. The early B-V color of the models is much too red at early phases compared to normal SNe Ia. This was also found for the models in Kromer et al. (2010). They argued that this was mainly due to blanketing effects of the burning products of the helium shell. The g-r color of Model M2a is also redder than SN 2016jhr (once corrected for redshift, see Fig. 10). The g-r color of SN 2018byg around maximum is very red, (De et al. (2019) found g-r $\approx 2$ mag at peak light). This is significantly redder than is seen for the angle averaged g-r color of Model M2a around maximum. The extreme redness near peak for this object is a result of the strong line blanketing seen in the spectra.

As discussed in Section 5.3, SN 2016jhr showed an optical flash and red color evolution at early times, and SN 2018byg showed a rapid early rise in the $r$ band. Noebauer et al. (2017) find that model FM3 produces an early peak in the $\mathrm{U}$ and $\mathrm{B}$ bands, and a pronounced shoulder in the $\mathrm{V}$ and $\mathrm{R}$ bands within the first two days from explosion, which is consistent with these observations. ARTIs, however, is ill suited for modeling such optically thick conditions at these very early times. The abundances listed in Table 2 indicate that Models M2a, M1a, and M2a_i55 have large abundances of radioactive material present in the outer ejecta produced by the helium shell detonation. As such, we would expect these models to also show an early peak in the light curves at early times due to radioactive decays. This effect should be investigated in future work.

The angle averaged spectrum of Model M2a at 2 days before maximum is compared to SN 2016jhr, SN 2018byg, and SN 2011fe at similar epochs, see Fig. 12. In order to compare spectral features we scale the spectra of SN 2016jhr and SN 2018byg to match the brightness of Model M2a, given that the light curves in Fig. 10 show that the absolute brightnesses of these objects at this epoch are not a close match to Model M2a. As could be anticipated from the light curve plots and previous studies (Kromer et al. 2010), the models are not a good match to SN $2011 \mathrm{fe}$. The spectrum does, however, show some characteristic features, such as the Si line.

Interestingly, SN 2016jhr does show similar features to Model M2a. Jiang et al. (2017) found prominent absorption features, such as the Ti II trough at $\approx 4150 \AA$. All of our models show similarly strong Ti II absorption. SN 2016jhr shows Ca II absorption around $\approx 3700 \AA$, comparable to Model M2a, and although we see weaker Si II absorption at $\approx 6100 \AA$ in Model $\mathrm{M} 2 \mathrm{a}$, the velocity of this feature is similar. While our models do 

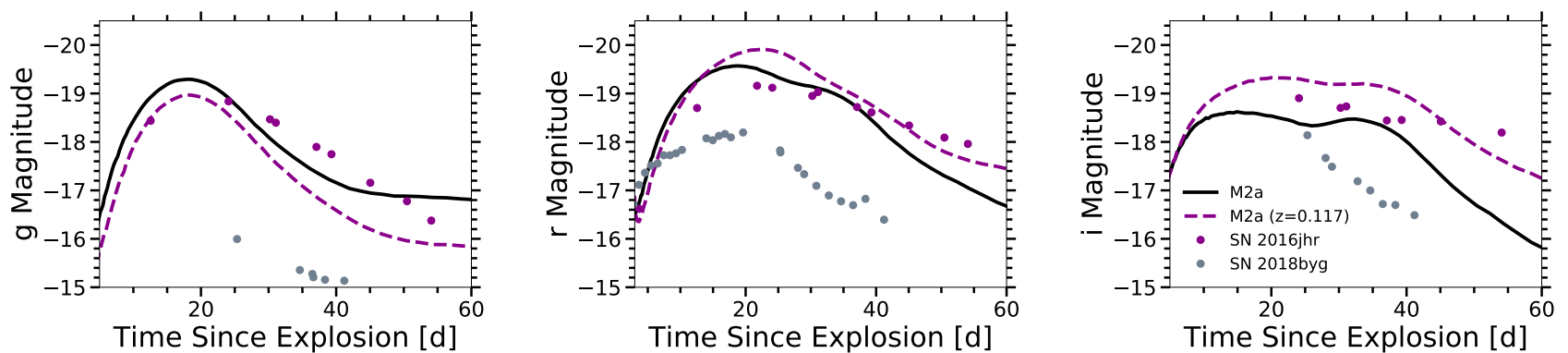

Fig. 10: Angle averaged g, r, and i band limited light curves of Model M2a compared to the light curves of SN $2016 \mathrm{jhr}$ (Jiang et al. 2017) and SN 2018byg (De et al. 2019). We also show the light curves of Model M2a as would be observed for an object at a redshift of $\mathrm{z}=0.11737$ (dashed purple line) to make a direct comparison to SN 2016jhr.
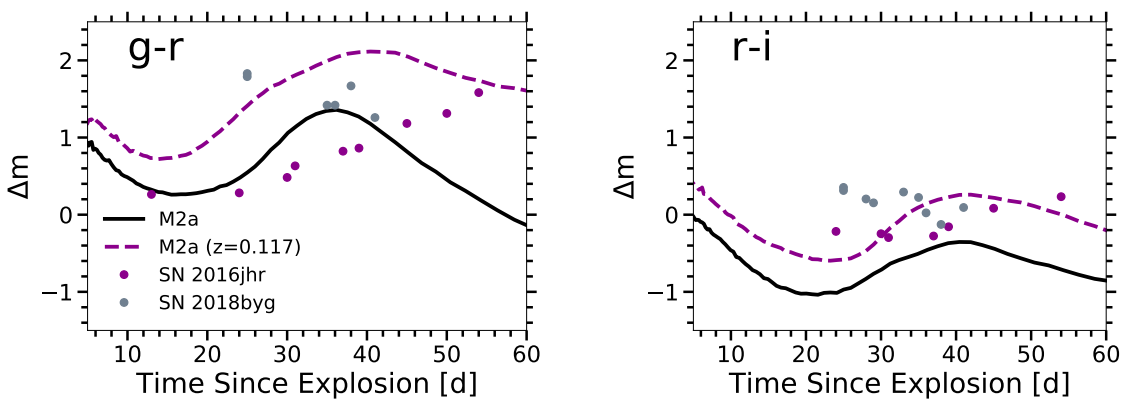

Fig. 11: Angle averaged g-r and r-i color curves for Model M2a compared to the colors of SN 2016jhr (Jiang et al. 2017) and SN 2018byg (De et al. 2019). We also plot Model M2a at redshift z=0.11737 for comparison with SN 2016jhr.

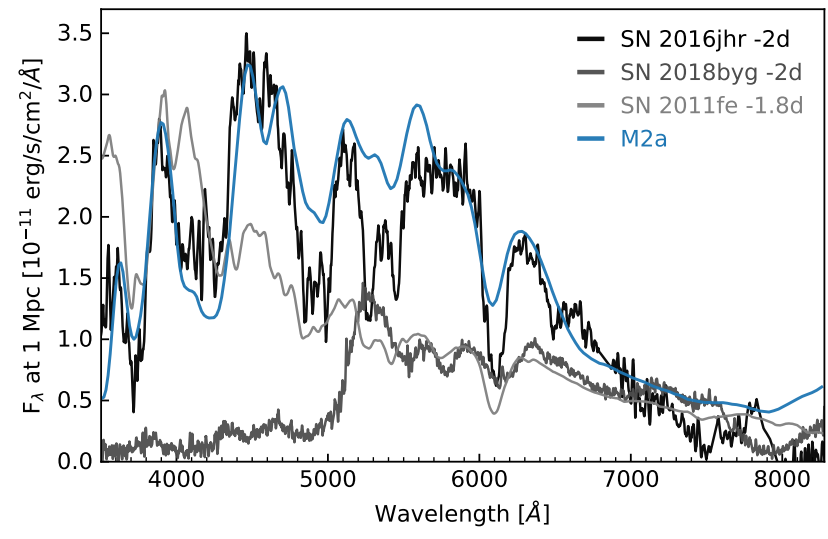

Fig. 12: Angle averaged spectrum of Model M2a at 15 days after explosion ( $\sim 2$ days before peak) compared to the spectra of SN 2016jhr (Jiang et al. 2017) 2 days before peak, SN 2018byg (De et al. 2019) 2 days before peak, and SN 2011fe (Nugent et al. 2011) 1.8 days before peak. SN 2016jhr and SN 2018byg have both been suggested to have been helium detonations. The spectra for SN 2016jhr and SN 2018byg have been de-reddened and redshift corrected, and are scaled to match the brightness of Model M2a.

show strong absorption features in the blue, and are a reasonable match to SN 2016jhr at 2 days before peak, the angle averaged properties are not able to account for the line blanketing seen in SN 2018 byg for wavelengths blueward of $\approx 5100 \AA$. We discuss the comparison to SN 2018byg further in Section 5.5.

\subsection{Viewing angle effects}

Kromer et al. (2010) show that an observer viewing the explosion from the polar directions observes a redder spectrum, or a bluer spectrum than viewing from equator on due to the asymmetrical distribution of iron-group elements produced in the outer layers of their models. It is interesting that we see more absorption is necessary for the models to account for the strong line blanketing of SN 2018byg, relative to the angle averaged spectrum, as this is similar to the differences presented by Kromer et al. (2010) between the equatorial line of sight spectrum, and the polar line of sight spectrum where the observer is looking through an extended layer of iron-group material produced in the helium detonation.

The off-center ignition of the scissors mechanism creates strong asymmetries in the ejecta. Fig. 13 shows $2 \mathrm{D}$ slices along the $x$-axis of Model M2a. The colors indicate the mass fraction for each of $\mathrm{He}, \mathrm{Si}, \mathrm{S}, \mathrm{Ca}, \mathrm{Ti}$, and Ni. Higher abundances of irongroup and intermediate mass elements are synthesized around the positive $z$-axis (see $\mathrm{Si}, \mathrm{S}, \mathrm{Ca}$ in Fig. 13). These asymmetries create strong viewing angle effects for this model. We find that the asymmetries in our Models M1a and M2a_i55 are similar to M2a. The viewing angle effects of Model FM3 are discussed by Kromer et al. (2010).

We show in Fig. 14 the angle dependent light curves in the $\mathrm{g}$ and $\mathrm{r}$ bands for model M2a, compared to the light curves of SN 2016jhr and SN 2018byg. The g band light curves are more strongly affected by the asymmetries in the ejecta, as we see a difference of $\sim 1$ mag in peak brightness between the angles presented here. It is apparent that the level of absorption viewed by an observer is a strong function of orientation. As discussed in Section 5.4 the degree of absorption in the angle averaged spec- 

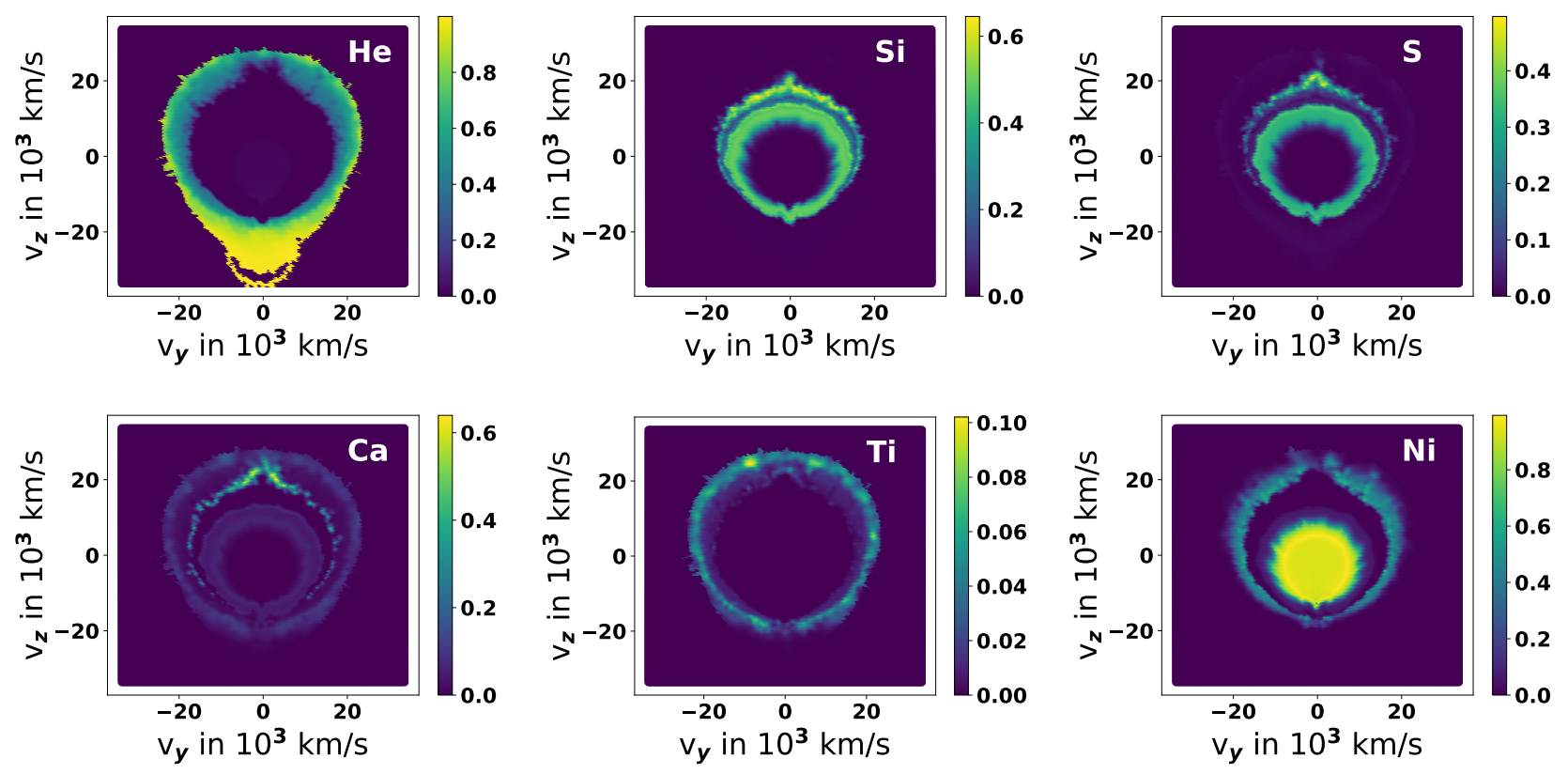

Fig. 13: Slice along X-axis of Model M2a showing abundances for specific elements, where the color bar shows the mass fraction for that element.

trum of our models is not sufficient to account for the level of line blanketing observed for SN 2018byg. However, the high degree of blanketing required by SN 2018byg is reproduced for the most extreme lines of sight, plotted in Fig. 15, which shows the viewing angle dependent spectra calculated for Model M2a at 12 days after explosion, and the spectrum of SN 2018byg at a similar epoch (13 days). The higher abundance of heavy elements (see Fig. 13) in the direction of an observer looking toward the helium ignition point on the positive $\mathrm{z}$-axis $\left(\theta=0^{\circ}\right)$ causes stronger absorption than for the angle averaged spectra, as is seen in Fig. 15. The spectrum viewed at $45^{\circ}$ is similar to $\theta=0^{\circ}$. These spectra also show strong line blanketing in the blue regions of the spectra, and deep Ca II absorption features. At $\theta=180^{\circ}$ significantly less absorption is seen due to the lower abundance of heavy elements. The spectrum seen at $\theta=90^{\circ}$ is similar to the angle averaged spectrum. These results highlight the necessity of multi-dimensional simulations.

\section{Conclusions}

In this work we describe a detonation ignition mechanism for the double detonation scenario that has previously not received much attention: the scissors mechanism. Most simulations carried out so far are in 1D or 2D (e.g., Woosley \& Weaver 1994; Bildsten et al. 2007) with only Moll \& Woosley (2013) performing 3D simulations. Here 3D simulations were carried out using the ArEpo code. Its adaptive mesh allows us to study the evolution of the helium shell detonation with high spatial resolution.

A detonation ignition mechanism is detected: The detonation and shock waves propagate in the helium shell and in the core. At the point when the detonation wave in the helium shell converges opposite to its ignition spot, high enough densities and temperatures are reached in a large enough volume to ignite a second detonation. This detonation propagates into the core and leads to its complete incineration. This core detonation ignition mechanism differs from the converging shock mechanism of Fink et al. (2007, 2010) and Moll \& Woosley (2013). In our models, we assumed a non-rotating progenitor configuration. Rotation introduces a symmetry axis and García-Senz et al. (2018) find that a He detonation ignition far from this axis blurs the convergence of the detonation wave on the antipode. They conclude, however, that an ignition of core detonation is still likely to occur. We note that if the scissors mechanism discussed here fails, the mechanism associated with the converging shock detonation would occur.

We find that the scissors mechanism is independent of the WD mass. However, the profile of the transition between $\mathrm{CO}$ core and He shell is important (see Sec. 4.3). A change in the mixing of carbon into the He shell can result in a different shell composition and detonation ignition mechanism.

The simulations show that the mechanism is robust for different resolutions and that the energy release is converged. However, as the carbon detonation cannot be fully resolved the carbon ignition is partly a numerical effect. Nevertheless, as critical values found in previous work (Röpke et al. 2007; Seitenzahl et al. 2009) are reached in more than one cell it is reasonable to say that the detonation is physical.

The final abundances from the helium detonation of Model M2a show differences of about one order of magnitude for many isotopes compared to FM3. The difference in the ${ }^{56} \mathrm{Ni}$ abundance in our Model M2a is a result of the different physical conditions in the setup and burning treatment. Röpke (2017) describes the details of the combustion processes. It should be noted that Fink et al. (2010) consider a different ignition mechanism for the second detonation and their simulations are in 2D. The final total ${ }^{56} \mathrm{Ni}$ abundance, however, is of the same order of magnitude and corresponds to a mass in the expected range for a $\mathrm{SN} \mathrm{Ia}$ (Stritzinger et al. 2006; Scalzo et al. 2014).

Radiative transfer calculations were carried out using the radiative transfer code ARTIs. We present the synthetic observables of Models M2a, M1a, and M2a_i55, and we compare these to Model FM3 and to observed SNe Ia. We find that despite the differences in the explosion models their light curves and spectra show no significant differences. While these models show some 

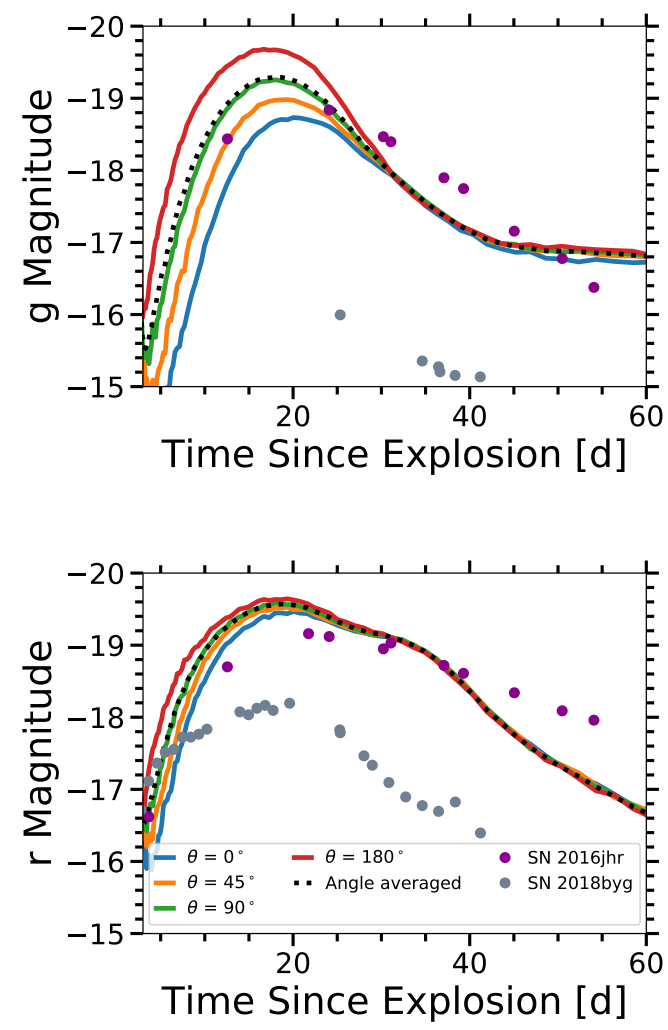

Fig. 14: Viewing angle dependent $\mathrm{g}$ and $\mathrm{r}$ band limited light curves for Model M2a, compared to the light curves for SN 2016jhr and SN 2018byg. The angle averaged light curves are plotted (dotted black line) for reference.

differences compared to Model FM3, these are small considering the apparent discrepancies with observations.

As was found by Kromer et al. (2010), our models are too red compared to observations of normal SNe Ia. This is particularly obvious in the B-V color, where our models are redder than the spectroscopically normal SN 2011fe at all epochs considered. Additionally, our models are only able to match some spectral features, such as the Si II line, typical for normal SNe Ia near maximum light.

We also compare our models to SN 2016jhr (Jiang et al. 2017) and SN 2018byg (De et al. 2019), which are unusual SNe Ia specifically suggested to have been triggered by helium shell detonations. We find that the g-r color of our models is redder than SN 2016jhr near maximum light, however, we find that the extreme redness of SN 2018byg around maximum is significantly redder than our models due to the line blanketing observed for SN 2018byg.

We find that the near maximum spectrum of SN 2016jhr is a reasonable match to the angle averaged spectrum of Model M2a, which is similar to the equatorial line of sight. In particular the strong absorption due to intermediate mass elements seen at the blue wavelengths of the spectrum is similar to that found for Model M2a. The angle averaged spectrum for Model M2a does not produce enough line blanketing to account for that seen in SN 2018byg, however, the most extreme lines of sight are able to reproduce this level of absorption at the blue end of the spectrum.

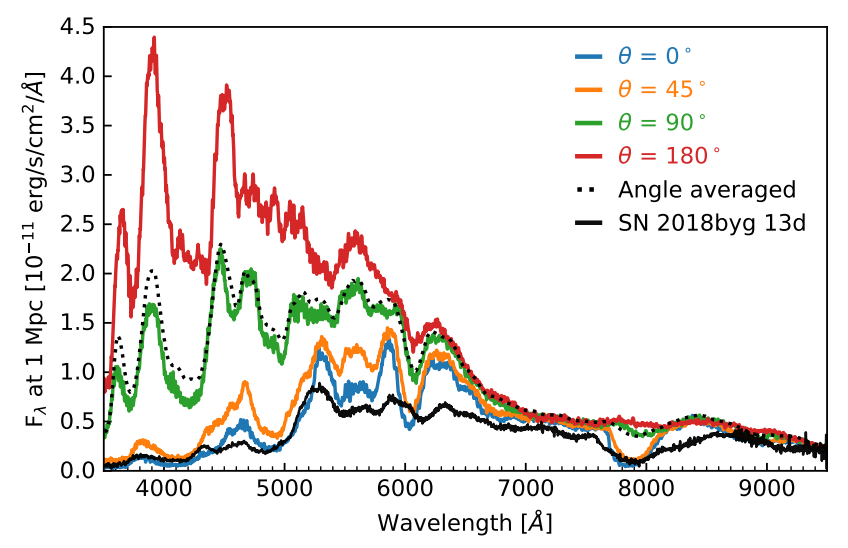

Fig. 15: Viewing angle dependent spectra for Model M2a at 12 days after explosion. We show the spectra for viewing angles of $\theta=0^{\circ}, 45^{\circ}, 90^{\circ}$, and $180^{\circ}$, and plot the angle averaged spectrum for comparison. Also plotted is the spectrum of SN 2018byg at a similar epoch (13 days after explosion). The spectrum of SN 2018byg has been de-reddened and redshift corrected.

Here we have proven that the amount of core-shell mixing is an important parameter which influences the details of the carbon detonation ignition. We find that the double detonation scenario includes a further carbon detonation ignition mechanism - namely the scissors mechanism - which did not receive much attention before and strongly depends on the amount of mixing. However, we have only investigated a limited set of parameters. Therefore it is necessary in the future to conduct a parameter study. The dependence of the final yields and stability of the detonation ignition mechanism on the mass of the helium shell and $\mathrm{CO}$ core, as well as metallicity and carbon abundance in the shell will be studied.

Acknowledgements. We thank our referee for insightful comments that helped to greatly improve this article. This work was supported by the Deutsche Forschungsgemeinschaft (DFG, German Research Foundation) - Project-ID 138713538 - SFB 881 ("The Milky Way System", subproject A10). SG, STO, RP, MK, and FKR acknowledge support by the Klaus Tschira Foundation. SAS acknowledges support form the UK STFC through grant ST/P000312/1. IRS was supported by the Australian Research Council through Grant FT160100028. NumPy and SciPy (Oliphant 2007), IPython (Pérez \& Granger 2007), and Matplotlib (Hunter 2007) were used for data processing and plotting. The authors gratefully acknowledge the Gauss Centre for Supercomputing e.V. (www.gauss-centre.eu) for funding this project by providing computing time on the GCS Supercomputer JUWELS (Jülich Supercomputing Centre 2019) at Jülich Supercomputing Centre (JSC). This work was performed using the DiRAC Data Intensive service at Leicester, operated by the University of Leicester IT Services, which forms part of the STFC DiRAC HPC Facility (www.dirac.ac.uk). The equipment was funded by BEIS capital funding via STFC capital grants ST/K000373/1 and ST/R002363/1 and STFC DiRAC Operations grant ST/R001014/1. This work also used the Cambridge Service for Data Driven Discovery (CSD3), part of which is operated by the University of Cambridge Research Computing on behalf of the STFC DiRAC HPC Facility (www.dirac.ac.uk). The DiRAC component of CSD3 was funded by BEIS capital funding via STFC capital grants ST/P002307/1 and ST/R002452/1 and STFC operations grant ST/R00689X/1. DiRAC is part of the National e-Infrastructure. This work was supported by computational resources provided by the Australian Government through the National Computational Infrastructure (NCI) under the National Computational Merit Allocation Scheme.

\section{References}

Arnett, W. D. 1969, Ap\&SS, 5, 180

Bildsten, L., Shen, K. J., Weinberg, N. N., \& Nelemans, G. 2007, ApJ, 662, L95 Botyánszki, J., Kasen, D., \& Plewa, T. 2018, ApJ, 852, L6

Boyle, A., Sim, S. A., Hachinger, S., \& Kerzendorf, W. 2017, A\&A, 599, A46 
Bulla, M., Sim, S. A., \& Kromer, M. 2015, MNRAS, 450, 967

Cyburt, R. H., Amthor, A. M., Ferguson, R., et al. 2010, The Astrophysical Journal Supplement Series, 189, 240

De, K., Kasliwal, M. M., Polin, A., et al. 2019, The Astrophysical Journal, 873, L18

Fink, M., Hillebrandt, W., \& Röpke, F. K. 2007, A\&A, 476, 1133

Fink, M., Kromer, M., Seitenzahl, I. R., et al. 2014, MNRAS, 438, 1762

Fink, M., Röpke, F. K., Hillebrandt, W., et al. 2010, A\&A, 514, A53

Forcada, R. 2008, Proceedings of Science, PoS(SUPERNOVA)012

Forcada, R., Garcia-Senz, D., \& José, J. 2006, in International Symposium on Nuclear Astrophysics - Nuclei in the Cosmos

Fryxell, B. A., Müller, E., \& Arnett, W. D. 1989, Hydrodynamics and nuclear burning, MPA Green Report 449, Max-Planck-Institut für Astrophysik, Garching

Fynbo, H. O. U., Diget, C. A., Bergmann, U. C., et al. 2005, Nature, 433, 136

Gall, E. E. E., Taubenberger, S., Kromer, M., et al. 2012, MNRAS, 427, 994

García-Senz, D., Bravo, E., \& Woosley, S. E. 1999, A\&A, 349, 177

García-Senz, D., Cabezón, R. M., \& Domínguez, I. 2018, ApJ, 862, 27

Gilfanov, M. \& Bogdán, Á. 2010, Nature, 463, 924

Glasner, A., Livne, E., Steinberg, E., Yalinewich, A., \& Truran, J. W. 2018, MNRAS, 476, 2238

Górski, K. M., Hivon, E., Banday, A. J., et al. 2005, ApJ, 622, 759

Guillochon, J., Dan, M., Ramirez-Ruiz, E., \& Rosswog, S. 2010, ApJ, 709, L64

Hunter, J. D. 2007, Computing in Science \& Engineering, 9, 90

Iben, Jr., I. \& Tutukov, A. V. 1984, ApJS, 54, 335

Jacobs, A. M., Zingale, M., Nonaka, A., Almgren, A. S., \& Bell, J. B. 2016, ApJ, 827,84

Jiang, J.-A., Doi, M., Maeda, K., et al. 2017, Nature, 550, 80

Jülich Supercomputing Centre. 2019, Journal of large-scale research facilities, 5

Katz, M. P. \& Zingale, M. 2019, ApJ, 874, 169

Kromer, M., Ohlmann, S., \& Röpke, F. K. 2017, Mem. Soc. Astron. Italiana, 88, 312

Kromer, M. \& Sim, S. A. 2009, MNRAS, 398, 1809

Kromer, M., Sim, S. A., Fink, M., et al. 2010, ApJ, 719, 1067

Livne, E. 1990, ApJ, 354, L53

Livne, E. 1997, in NATO ASIC Proc. 486: Thermonuclear Supernovae, ed. P. Ruiz-Lapuente, R. Canal, \& J. Isern, 425-440

Livne, E. \& Arnett, D. 1995, ApJ, 452, 62

Livne, E. \& Glasner, A. S. 1990, ApJ, 361, 244

Livne, E. \& Glasner, A. S. 1991, ApJ, 370, 272

Lucy, L. B. 2002, A\&A, 384, 725

Lucy, L. B. 2003, A\&A, 403, 261

Lucy, L. B. 2005, A\&A, 429, 19

Maoz, D., Mannucci, F., \& Nelemans, G. 2014, ARA\&A, 52, 107

Moll, R. \& Woosley, S. E. 2013, ApJ, 774, 137

Neunteufel, P., Yoon, S. C., \& Langer, N. 2017, A\&A, 602, A55

Noebauer, U. M., Kromer, M., Taubenberger, S., et al. 2017, MNRAS, 472, 2787

Nomoto, K. 1982, ApJ, 257, 780

Nugent, P. E., Sullivan, M., Cenko, S. B., et al. 2011, Nature, 480, 344

Ohlmann, S. T., Röpke, F. K., Pakmor, R., \& Springel, V. 2017, A\&A, 599, A5

Oliphant, T. E. 2007, Computing in Science \& Engineering, 9, 10

Pakmor, R., Edelmann, P., Röpke, F. K., \& Hillebrandt, W. 2012, MNRAS, 424, 2222

Pakmor, R., Hachinger, S., Röpke, F. K., \& Hillebrandt, W. 2011, A\&A, 528, A117+

Pakmor, R., Kromer, M., Röpke, F. K., et al. 2010, Nature, 463, 61

Pakmor, R., Kromer, M., Taubenberger, S., \& Springel, V. 2013, ApJ, 770, L8

Pakmor, R., Springel, V., Bauer, A., et al. 2016, MNRAS, 455, 1134

Pérez, F. \& Granger, B. E. 2007, Computing in Science \& Engineering, 9, 21

Rauscher, T. \& Thielemann, F.-K. 2000, Atomic Data and Nuclear Data Tables, 75,1

Reinecke, M., Hillebrandt, W., \& Niemeyer, J. C. 2002, A\&A, 391, 1167

Röpke, F. K. 2017, in Handbook of Supernovae, ed. A. Alsabti \& P. Murdin (Springer), 1185-1209

Röpke, F. K., Woosley, S. E., \& Hillebrandt, W. 2007, ApJ, 660, 1344

Scalzo, R., Aldering, G., Antilogus, P., et al. 2014, MNRAS, 440, 1498

Seitenzahl, I. R., Cescutti, G., Röpke, F. K., Ruiter, A. J., \& Pakmor, R. 2013a, A\&A, 559, L5

Seitenzahl, I. R., Ciaraldi-Schoolmann, F., Röpke, F. K., et al. 2013b, MNRAS, 429, 1156

Seitenzahl, I. R., Meakin, C. A., Townsley, D. M., Lamb, D. Q., \& Truran, J. W. 2009, ApJ, 696, 515

Shen, K. J. \& Bildsten, L. 2014, ApJ, 785, 61

Shen, K. J., Boubert, D., Gänsicke, B. T., et al. 2018, ApJ, 865, 15

Shen, K. J. \& Moore, K. 2014, ApJ, 797, 46

Shigeyama, T., Nomoto, K., Yamaoka, H., \& Thielemann, F. 1992, ApJ, 386, L13

Sim, S. A. 2007, MNRAS, 375, 154

Sim, S. A., Fink, M., Kromer, M., et al. 2012, MNRAS, 420, 3003

Sim, S. A., Röpke, F. K., Hillebrandt, W., et al. 2010, ApJ, 714, L52

Article number, page 18 of 18
Sim, S. A., Seitenzahl, I. R., Kromer, M., et al. 2013, MNRAS, 436, 333

Springel, V. 2010, MNRAS, 401, 791

Stritzinger, M., Mazzali, P. A., Sollerman, J., \& Benetti, S. 2006, A\&A, 460, 793

Timmes, F. X. \& Swesty, F. D. 2000, ApJS, 126, 501

Townsley, D. M., Miles, B. J., Shen, K. J., \& Kasen, D. 2019, ApJ, 878, L38

Townsley, D. M., Miles, B. J., Timmes, F. X., Calder, A. C., \& Brown, E. F. 2016, ApJS, 225, 3

Townsley, D. M., Moore, K., \& Bildsten, L. 2012, ApJ, 755, 4

Travaglio, C., Hillebrandt, W., Reinecke, M., \& Thielemann, F.-K. 2004, A\&A, 425, 1029

Waldman, R., Sauer, D., Livne, E., et al. 2011, ApJ, 738, 21

Wang, X., Wang, L., Filippenko, A. V., et al. 2012, ApJ, 749, 126

Wilk, K. D., Hillier, D. J., \& Dessart, L. 2018, MNRAS, 474, 3187

Woosley, S. E. \& Kasen, D. 2011, ApJ, 734, 38

Woosley, S. E. \& Weaver, T. A. 1994, in Les Houches Session LIV: Supernovae, ed. S. A. Bludman, R. Mochkovitch, \& J. Zinn-Justin (Amsterdam: NorthHolland), 63-154

Xu, Y., Takahashi, K., Goriely, S., et al. 2013, Nucl. Phys. A, 918, 61 